\title{
ASYMPTOTIC BEHAVIOR OF THE NUMERICAL SOLUTIONS OF TIME-DELAYED REACTION DIFFUSION EQUATIONS WITH NON-MONOTONE REACTION TERM*
}

\author{
YUAN-MING WANG ${ }^{1}$
}

\begin{abstract}
This paper is concerned with the asymptotic behavior of the finite difference solutions of a class of nonlinear reaction diffusion equations with time delay. By introducing a pair of coupled upper and lower solutions, an existence result of the solution is given and an attractor of the solution is obtained without monotonicity assumptions on the nonlinear reaction function. This attractor is a sector between two coupled quasi-solutions of the corresponding "steady-state" problem, which are obtained from a monotone iteration process. A sufficient condition, ensuring that two coupled quasisolutions coincide, is given. Also given is the application to a nonlinear reaction diffusion problem with time delay for three different types of reaction functions, including some numerical results which validate the theoretical analysis.
\end{abstract}

Mathematics Subject Classification. 35K57, 65M06, 74H40.

Received: January 23, 2002. Revised: November 20, 2002.

\section{INTRODUCTION}

In the study of various types of nonlinear reaction diffusion problems, a major concern is the asymptotic behavior of the time-dependent solution in relation to the solution of the corresponding steady-state problem (see $[4,7,14,18]$ ). In particular, the information about the asymptotic behavior of the time-dependent solution is very important for the study of the coexistence and persistence problems to certain biological and ecological systems with or without time delays (see $[6,8,19]$ ). Recently, many studies have been given to the numerical simulation for the asymptotic behavior of the time-dependent solution (see $[10,11]$ ). In this paper, we consider

Keywords and phrases. Asymptotic behavior, finite difference equation, reaction diffusion equation, time delay, upper and lower solutions.

* This work was partially supported by the National Natural Science Foundation of China under Grant No.10001012, the Youth Science Foundation of Shanghai Higher Education under Grant No.2000QN15, and Shanghai Priority Academic Discipline. Part of the work was completed during the author's visit to CMAP of École Polytechnique in France.

${ }^{1}$ Department of Mathematics, East China Normal University, Shanghai 200062, China. e-mail: ymwang@math.ecnu.edu.cn 
the following reaction diffusion problem with time delay:

$$
\left\{\begin{array}{l}
\frac{\partial u}{\partial t}-\nabla \cdot(D(x) \nabla u)=f\left(x, u, u_{\tau}\right), \quad x \in \Omega, t>0 \\
\alpha(x) \frac{\partial u}{\partial \nu}+\beta(x) u=g(x), \quad x \in \partial \Omega, t>0 \\
u(x, t)=\psi(x, t), \quad x \in \Omega,-\tau \leq t \leq 0
\end{array}\right.
$$

where $\Omega$ is a bounded domain in $\mathbb{R}^{p}$ with the boundary $\partial \Omega(p=1,2, \ldots), \frac{\partial}{\partial \nu}$ denotes the outward normal derivative on $\partial \Omega$, and $u_{\tau} \equiv u(x, t-\tau)$ for a given $\tau>0$. It is assumed that the function $D(x)$ is a positive function in $\bar{\Omega}=\Omega \cup \partial \Omega$, and $\alpha(x) \geq 0, \beta(x) \geq 0$ with $\alpha(x)+\beta(x)>0$ on $\partial \Omega$. The latter assumption includes the Dirichlet boundary condition $(\alpha(x) \equiv 0, \beta(x) \equiv 1)$, Neumann boundary condition $(\alpha(x) \equiv 1, \beta(x) \equiv 0)$, and Robin boundary condition $(\alpha(x) \equiv 1, \beta(x) \geq 0)$. It is also assumed that all the prescribed functions $f$, $g$, and $\psi$ in (1.1) are continuous in their respective domains, and the function $\psi(x, t)$ satisfies the boundary condition for $-\tau \leq t \leq 0$. The function $f\left(\cdot, u, u_{\tau}\right)$ is, in general, nonlinear in $u$ and $u_{\tau}$. The purpose of this paper is to investigate the asymptotic behavior of the finite difference solution for the above model problem in relation to the finite difference solution of its corresponding steady-state problem which is given by

$$
\left\{\begin{array}{l}
-\nabla \cdot(D(x) \nabla u)=f(x, u, u), \quad x \in \Omega \\
\alpha(x) \frac{\partial u}{\partial \nu}+\beta(x) u=g(x), \quad x \in \partial \Omega
\end{array}\right.
$$

Let $k$ be the time increment such that $s=\tau / k$ is a positive integer, and $h_{i}(i=1,2, \ldots, p)$ be the space increment. We assume that the domain $\bar{\Omega}$ is connected, and let $(\cdot)^{T}$ denote the transpose of a row vector. By applying the implicit method for parabolic equation and using a suitable finite difference approximation for the diffusion operator $\mathcal{L} u \equiv-\nabla \cdot(D(x) \nabla u)$ and the boundary operator $\mathcal{B} u \equiv \alpha(x) \frac{\partial u}{\partial \nu}+\beta(x) u$, we obtain a finite difference approximation of (1.1) in the form:

$$
\left\{\begin{array}{l}
(I+k A) \mathbf{u}_{n}=\mathbf{u}_{n-1}+k \mathbf{f}\left(\mathbf{u}_{n}, \mathbf{u}_{n-s}\right), \quad n=1,2, \ldots \\
\mathbf{u}_{n}=\Psi_{n}, \quad n=-s,-s+1, \ldots, 0
\end{array}\right.
$$

where $\mathbf{u}_{n} \equiv\left(u_{1, n}, \ldots, u_{N, n}\right)^{T}$ represents the solution vector at the $N$-mesh points $x_{i}$ in $\Omega$, and $A$ is an $N$ by $N$ matrix associated with the diffusion operator $\mathcal{L}$ and the boundary operator $\mathcal{B}$. The vectors $\Psi_{n} \in \mathbb{R}^{N}$, $n=-s, \ldots, 0$, are associated with the initial data in (1.1), and the vector function $\mathbf{f}\left(\mathbf{u}_{n}, \mathbf{u}_{n-s}\right)$ is given by

$$
\mathbf{f}\left(\mathbf{u}_{n}, \mathbf{u}_{n-s}\right) \equiv\left(f\left(x_{1}, u_{1, n}, u_{1, n-s}\right), \ldots, f\left(x_{N}, u_{N, n}, u_{N, n-s}\right)\right)^{T}+\mathbf{g}
$$

where $\mathbf{g}$ is associated with the boundary data in (1.1). Since our concern is the basic mathematical structure of the finite difference approximation (1.3), detailed formulation of the system (1.3) is omitted (see [1,16] for some detailed discussions).

Just as in the continuous problem, the finite difference approximation of (1.2) is given by

$$
A \mathbf{u}=\mathbf{f}(\mathbf{u}, \mathbf{u})
$$

where $\mathbf{u}=\left(u_{1}, \ldots, u_{N}\right)^{T}$ is the solution vector, and $\mathbf{f}(\mathbf{u}, \mathbf{u})$ is given by

$$
\mathbf{f}(\mathbf{u}, \mathbf{u}) \equiv\left(f\left(x_{1}, u_{1}, u_{1}\right), \ldots, f\left(x_{N}, u_{N}, u_{N}\right)\right)^{T}+\mathbf{g}
$$

Using the method of upper and lower solutions, Pao [13] gave two monotone iterative schemes for solving the system (1.3) when the reaction function $f\left(\cdot, u, u_{\tau}\right)$ is the sum of a monotone nondecreasing and a monotone 
nonincreasing function of $u_{\tau}$. An extension of these monotone iterative schemes to the general case was given in [12], where $f\left(\cdot, u, u_{\tau}\right)$ is not necessarily monotone in $u_{\tau}$. In [11], it was shown that for one class of initial vectors $\boldsymbol{\Psi}_{n}$ the solution $\mathbf{u}_{n}$ of the system (1.3) converges to the maximal solution of the system (1.4) as $n \rightarrow \infty$, while for another class of initial vectors $\boldsymbol{\Psi}_{n}$ it converges to the minimal solution. This convergence result describes the asymptotic behavior of the solution of the system (1.3) when time tends to infinity. However, it is only for the system $(1.3)$ where $f\left(\cdot, u, u_{\tau}\right)$ is monotone nondecreasing in $u_{\tau}$. This certainly limits its applications since the function $f$ may not possess any monotone property in practical problems. Thus we were motivated to give a qualitative description of the asymptotic behavior of the solution for the system (1.3) without monotonicity assumptions on $f$. This paper is to report our work in this effort.

The method of upper and lower solutions is a fundamental approach to investigate the asymptotic behavior of the solutions of reaction diffusion problems both analytically and numerically $(c f .[10,11,19])$. Although the method of upper and lower solutions in [12] leads to a computational algorithm that is applicable to a much larger class of systems in the form (1.3), it is difficult to use it to investigate the asymptotic behavior of the solution of the system (1.3) without monotonicity assumptions on $f$. In this paper, we firstly introduce a new concept of the coupled upper and lower solutions, and then use this new concept with an embedding technique to achieve the goal of this paper.

The rest of the paper is organized as follows. In Section 2, we introduce the concept of the coupled upper and lower solutions and then study the existence of the solutions of the system (1.3). Section 3 is devoted to the asymptotic behavior of the solutions of the system (1.3). In the final section, we give an application to a reaction diffusion problem with time delay for three different types of $f$, including some numerical results which validate the theoretical analysis.

\section{The EXISTENCE OF TIME-DEPENDENT SOLUTiOns}

Motivated by the reaction diffusion problem (1.1), we impose the following basic hypothesis on the matrix $A$.

$(\mathcal{H})$ The matrix $A=\left(a_{i, j}\right)$ is irreducible, and

$$
\left\{\begin{array}{l}
a_{i, i}>0, \quad i=1,2, \ldots, N, \quad a_{i, j} \leq 0, \quad i, j=1,2, \ldots, N \text { and } i \neq j, \\
\sum_{j=1}^{N} a_{i, j} \geq 0, \quad i=1,2, \ldots, N
\end{array}\right.
$$

Hypothesis $(\mathcal{H})$ implies that for any nonnegative but non-zero diagonal matrix $D$, the inverse matrix $(A+D)^{-1}$ exists and is a nonnegative matrix (see [3,17]). In fact, if the strict inequality $\sum_{j=1}^{N} a_{i, j}>0$ holds for at least one $i$ (which can always be satisfied when the boundary condition is not of Neumann type), then the inverse $A^{-1}$ exists and is a nonnegative matrix. The connectedness assumption of $\bar{\Omega}$ ensures that $A$ is irreducible, while the relation (2.1) can always be satisfied by a suitable finite difference approximation of the operators $\mathcal{L}$ and $\mathcal{B}$ (see $[5,16]$ for some detailed discussions).

Without further mention, we assume that all inequalities involving vectors are component-wise. Let $\mathbf{u}, \mathbf{v}$ and $\mathbf{w}$ be three vectors of the same dimension. If $\mathbf{u} \leq \mathbf{w} \leq \mathbf{v}$, we say that $\mathbf{w} \in \mathbf{K}(\mathbf{u}, \mathbf{v})$.

To investigate the asymptotic behavior of the solution of the system (1.3) without monotonicity assumptions on $f$, we need to a pair of the coupled upper and lower solutions as follows.

Definition 2.1. Two sequences of vectors $\overline{\mathbf{u}}_{n}, \underline{\mathbf{u}}_{n} \in \mathbb{R}^{N}(n \geq-s)$ are called a pair of coupled upper and lower solutions of the system (1.3) if

(i) $\overline{\mathbf{u}}_{n} \geq \underline{\mathbf{u}}_{n}, \quad n=-s,-s+1, \ldots$;

(ii) there exists a nonnegative diagonal matrix $C_{n}$ such that for all $\mathbf{w}_{n} \in \mathbf{K}\left(\underline{\mathbf{u}}_{n}, \overline{\mathbf{u}}_{n}\right)$,

$$
\mathbf{f}\left(\mathbf{w}_{n}, \mathbf{u}_{n-s}\right)-\mathbf{f}\left(\mathbf{w}_{n}, \mathbf{v}_{n-s}\right) \geq-C_{n}\left(\mathbf{u}_{n-s}-\mathbf{v}_{n-s}\right), \quad n=1,2, \ldots
$$

whenever $\underline{\mathbf{u}}_{n-s} \leq \mathbf{v}_{n-s} \leq \mathbf{u}_{n-s} \leq \overline{\mathbf{u}}_{n-s}$; 
(iii) for the nonnegative diagonal matrix $C_{n}$ in $(2.2)$,

$$
\begin{cases}(I+k A) \overline{\mathbf{u}}_{n} \geq \overline{\mathbf{u}}_{n-1}+k\left(\mathbf{f}\left(\overline{\mathbf{u}}_{n}, \overline{\mathbf{u}}_{n-s}\right)+C_{n}\left(\overline{\mathbf{u}}_{n-s}-\underline{\mathbf{u}}_{n-s}\right)\right), & n=1,2, \ldots, \\ (I+k A) \underline{\mathbf{u}}_{n} \leq \underline{\mathbf{u}}_{n-1}+k\left(\mathbf{f}\left(\underline{\mathbf{u}}_{n}, \underline{\mathbf{u}}_{n-s}\right)-C_{n}\left(\overline{\mathbf{u}}_{n-s}-\underline{\mathbf{u}}_{n-s}\right)\right), & n=1,2, \ldots, \\ \overline{\mathbf{u}}_{n} \geq \boldsymbol{\Psi}_{n} \geq \underline{\mathbf{u}}_{n}, \quad n=-s,-s+1, \ldots, 0 . & \end{cases}
$$

Remark 2.1. The above definition does not require the monotone property of the function $f\left(\cdot, u, u_{\tau}\right)$ in $u_{\tau}$. If $f\left(\cdot, u, u_{\tau}\right)$ is monotone nondecreasing in $u_{\tau}$, we take $C_{n}=0$. In this case, the above definition is reduced to usual ones (see, e.g., Def. 2.1 in [13]). If $f\left(\cdot, u, u_{\tau}\right)$ is monotone nonincreasing in $u_{\tau}$, the coupled upper and lower solutions here are also the coupled upper and lower solutions in [13] (see Def. 3.1 in [13]).

Remark 2.2. To develop a monotone iterative scheme for the system (1.3) without monotonicity assumptions on $f$, Pao [12] also gave a restricted definition of the coupled upper and lower solutions. It can be easily shown that a pair of coupled upper and lower solutions here is also a pair of coupled upper and lower solutions in [12]. This means that a pair of coupled upper and lower solutions here is more restrictive than that in [12]. However, this restrictive version is still useful. Our primary interest in it is hopefully to use it as a tool to investigate the asymptotic behavior of the solution of the system (1.3) without monotonicity assumptions on $f$. On the other hand, the construction of the coupled upper and lower solutions defined here is not more complicated than the construction of that in [12]. Indeed, it can be easily implemented in some specific problems. In Section 4, we give some such examples where the coupled upper and lower solutions can be explicitly constructed even if $f$ dose not possess any monotone property.

We now turn to the main result of this section.

Theorem 2.1. Let the sequences of vectors $\overline{\mathbf{u}}_{n}$ and $\underline{\mathbf{u}}_{n}(n \geq-s)$ be a pair of coupled upper and lower solutions of the system (1.3), and assume that there exists a nonnegative diagonal matrix $\Gamma_{n}$ such that for all $\mathbf{w}_{n-s} \in$ $\mathbf{K}\left(\underline{\mathbf{u}}_{n-s}, \overline{\mathbf{u}}_{n-s}\right)$,

$$
\mathbf{f}\left(\mathbf{u}_{n}, \mathbf{w}_{n-s}\right)-\mathbf{f}\left(\mathbf{v}_{n}, \mathbf{w}_{n-s}\right) \geq-\Gamma_{n}\left(\mathbf{u}_{n}-\mathbf{v}_{n}\right), \quad n=1,2, \ldots
$$

whenever $\underline{\mathbf{u}}_{n} \leq \mathbf{v}_{n} \leq \mathbf{u}_{n} \leq \overline{\mathbf{u}}_{n}$. Then the system (1.3) has at least one solution $\mathbf{u}_{n}^{*} \in \mathbf{K}\left(\underline{\mathbf{u}}_{n}, \overline{\mathbf{u}}_{n}\right)$.

Proof. For any $\mathbf{u}_{1} \in \mathbf{K}\left(\underline{\mathbf{u}}_{1}, \overline{\mathbf{u}}_{1}\right)$, we consider the following problem:

$$
\left\{\begin{array}{l}
\left(I+k A+k \Gamma_{1}\right) \mathbf{v}_{1}=\mathbf{u}_{0}^{*}+k\left(\Gamma_{1} \mathbf{u}_{1}+\mathbf{f}\left(\mathbf{u}_{1}, \mathbf{u}_{1-s}^{*}\right)\right) \\
\mathbf{u}_{n}^{*}=\mathbf{\Psi}_{n}, \quad n=-s,-s+1, \ldots, 0
\end{array}\right.
$$

Since the inverse $\left(I+k A+k \Gamma_{1}\right)^{-1}$ exists, the above problem has the unique solution $\mathbf{v}_{1}$. Now we define the $\operatorname{map} \mathcal{T}_{1}: \mathbf{K}\left(\underline{\mathbf{u}}_{1}, \overline{\mathbf{u}}_{1}\right) \longrightarrow \mathbb{R}^{N}$ as

$$
\mathcal{T}_{1} \mathbf{u}_{1}=\mathbf{v}_{1}, \quad \forall \mathbf{u}_{1} \in \mathbf{K}\left(\underline{\mathbf{u}}_{1}, \overline{\mathbf{u}}_{1}\right) .
$$

We first show that $\mathbf{v}_{1} \in \mathbf{K}\left(\underline{\mathbf{u}}_{1}, \overline{\mathbf{u}}_{1}\right)$. Let $\mathbf{w}_{1}=\mathbf{v}_{1}-\underline{\mathbf{u}}_{1}$. Then by (2.5) and (2.3) with $n=1$,

$$
\left(I+k A+k \Gamma_{1}\right) \mathbf{w}_{1} \geq k\left(\Gamma_{1} \mathbf{u}_{1}+\mathbf{f}\left(\mathbf{u}_{1}, \mathbf{u}_{1-s}^{*}\right)-\Gamma_{1} \underline{\mathbf{u}}_{1}-\mathbf{f}\left(\underline{\mathbf{u}}_{1}, \underline{\mathbf{u}}_{1-s}\right)+C_{1}\left(\overline{\mathbf{u}}_{1-s}-\underline{\mathbf{u}}_{1-s}\right)\right) .
$$

Because $\mathbf{u}_{1} \in \mathbf{k}\left(\underline{\mathbf{u}}_{1}, \overline{\mathbf{u}}_{1}\right)$, we have from (2.4) and (2.2) with $n=1$ that

$$
\begin{aligned}
\left(I+k A+k \Gamma_{1}\right) \mathbf{w}_{1} & \geq k\left(\mathbf{f}\left(\underline{\mathbf{u}}_{1}, \mathbf{u}_{1-s}^{*}\right)-\mathbf{f}\left(\underline{\mathbf{u}}_{1}, \underline{\mathbf{u}}_{1-s}\right)+C_{1}\left(\overline{\mathbf{u}}_{1-s}-\underline{\mathbf{u}}_{1-s}\right)\right) \\
& \geq k\left(-C_{1}\left(\mathbf{u}_{1-s}^{*}-\underline{\mathbf{u}}_{1-s}\right)+C_{1}\left(\overline{\mathbf{u}}_{1-s}-\underline{\mathbf{u}}_{1-s}\right)\right) \\
& \geq \mathbf{0} .
\end{aligned}
$$


Due to $\left(I+k A+k \Gamma_{1}\right)^{-1} \geq 0$, we obtain $\mathbf{w}_{1} \geq \mathbf{0}$ which implies $\mathbf{v}_{1} \geq \underline{\mathbf{u}}_{1}$. A similar argument shows $\mathbf{v}_{1} \leq \overline{\mathbf{u}}_{1}$. This proves that $\mathbf{v}_{1} \in \mathbf{K}\left(\underline{\mathbf{u}}_{1}, \overline{\mathbf{u}}_{1}\right)$. It is clear that $\mathcal{T}_{1}$ is a continuous map from $\mathbf{K}\left(\underline{\mathbf{u}}_{1}, \overline{\mathbf{u}}_{1}\right)$ into itself because of the continuity of the function $f\left(\cdot, u, u_{\tau}\right)$. Thus by Brouwer's fixed point theorem, $\mathcal{T}_{1}$ has at least one fixed point $\mathbf{u}_{1}^{*} \in \mathbf{K}\left(\underline{\mathbf{u}}_{1}, \overline{\mathbf{u}}_{1}\right)$ and it satisfies

$$
\left\{\begin{array}{l}
(I+k A) \mathbf{u}_{1}^{*}=\mathbf{u}_{0}^{*}+k \mathbf{f}\left(\mathbf{u}_{1}^{*}, \mathbf{u}_{1-s}^{*}\right) \\
\mathbf{u}_{n}^{*}=\mathbf{\Psi}_{n}, \quad n=-s,-s+1, \ldots, 0
\end{array}\right.
$$

Using $\mathbf{u}_{n}^{*}(n=-s,-s+1, \ldots, 0,1)$ we define the map $\mathcal{T}_{2}: \mathbf{K}\left(\underline{\mathbf{u}}_{2}, \overline{\mathbf{u}}_{2}\right) \longrightarrow \mathbb{R}^{N}$ as

$$
\mathcal{T}_{2} \mathbf{u}_{2}=\mathbf{v}_{2}, \quad \forall \mathbf{u}_{2} \in \mathbf{K}\left(\underline{\mathbf{u}}_{2}, \overline{\mathbf{u}}_{2}\right)
$$

where $\mathbf{v}_{2}$ is defined as the unique solution of the problem:

$$
\left(I+k A+k \Gamma_{2}\right) \mathbf{v}_{2}=\mathbf{u}_{1}^{*}+k\left(\Gamma_{2} \mathbf{u}_{2}+\mathbf{f}\left(\mathbf{u}_{2}, \mathbf{u}_{2-s}^{*}\right)\right) .
$$

By the similar argument as that for $\mathcal{T}_{1}$, we conclude that $\mathcal{T}_{2}$ has at least one fixed point $\mathbf{u}_{2}^{*} \in \mathbf{K}\left(\underline{\mathbf{u}}_{2}, \overline{\mathbf{u}}_{2}\right)$ and it satisfies

$$
\left\{\begin{array}{l}
(I+k A) \mathbf{u}_{2}^{*}=\mathbf{u}_{1}^{*}+k \mathbf{f}\left(\mathbf{u}_{2}^{*}, \mathbf{u}_{2-s}^{*}\right) \\
\mathbf{u}_{n}^{*}=\mathbf{\Psi}_{n}, \quad n=-s,-s+1, \ldots, 0
\end{array}\right.
$$

A continuation of this process shows that there exists $\mathbf{u}_{n}^{*} \in \mathbf{K}\left(\underline{\mathbf{u}}_{n}, \overline{\mathbf{u}}_{n}\right)$ such that

$$
\left\{\begin{array}{l}
(I+k A) \mathbf{u}_{n}^{*}=\mathbf{u}_{n-1}^{*}+k \mathbf{f}\left(\mathbf{u}_{n}^{*}, \mathbf{u}_{n-s}^{*}\right), \quad n=1,2, \ldots, \\
\mathbf{u}_{n}^{*}=\mathbf{\Psi}_{n}, \quad n=-s,-s+1, \ldots, 0
\end{array}\right.
$$

This proves that $\mathbf{u}_{n}^{*}$ is a solution of the system (1.3) in $\mathbf{K}\left(\underline{\mathbf{u}}_{n}, \overline{\mathbf{u}}_{n}\right)$. The proof is completed.

Remark 2.3. (a) Theorem 2.1 shows that if the sequences of the vectors $\overline{\mathbf{u}}_{n}$ and $\underline{\mathbf{u}}_{n}(n \geq-s)$ are the coupled upper and lower solutions of the system (1.3), then the system (1.3) has at least one solution $\mathbf{u}_{n}^{*}$ under the local Lipschitz condition (2.4). Moreover, $\overline{\mathbf{u}}_{n}$ and $\underline{\mathbf{u}}_{n}$ serve as the upper and lower bounds of $\mathbf{u}_{n}^{*}$. Since $\overline{\mathbf{u}}_{n}$ and $\underline{\mathbf{u}}_{n}$ are also the coupled upper and lower solutions in [12], Theorem 2.1 in [12] also gives an existence result for the solution $\mathbf{u}_{n}^{*}$. However, this theorem as well as its proof are valid only when $\mathbf{f}(\mathbf{u}, \mathbf{v})$ is a $C^{1}$-function of $\mathbf{u}$ for $\mathbf{u} \in \mathbf{K}\left(\underline{\mathbf{u}}_{n}, \overline{\mathbf{u}}_{n}\right)$ and $\mathbf{v} \in \mathbf{K}\left(\underline{\mathbf{u}}_{n-s}, \overline{\mathbf{u}}_{n-s}\right)$, and the time increment $k$ satisfies the constraint condition:

$$
k \sigma_{n}<1, \quad n=1,2, \ldots
$$

where

$$
\sigma_{n}=\max \left\{\frac{\partial f}{\partial u}\left(x_{i}, u, v\right): \underline{u}_{i, n} \leq u \leq \bar{u}_{i, n} \text { and } \underline{u}_{i, n-s} \leq v \leq \bar{u}_{i, n-s}, \quad i=1, \ldots, N\right\} .
$$

In fact, if $\mathbf{f}(\mathbf{u}, \mathbf{v})$ is a $C^{1}$-function of $\mathbf{u}$ for $\mathbf{u} \in \mathbf{K}\left(\underline{\mathbf{u}}_{n}, \overline{\mathbf{u}}_{n}\right)$ and $\mathbf{v} \in \mathbf{K}\left(\underline{\mathbf{u}}_{n-s}, \overline{\mathbf{u}}_{n-s}\right)$, the local Lipschitz condition (2.4) is automatically satisfied with

$$
\Gamma_{n}=\operatorname{diag}\left(\gamma_{1, n}, \ldots, \gamma_{N, n}\right), \quad n=1,2, \ldots
$$


where $\gamma_{i, n}(i=1, \ldots, N ; n=1,2, \ldots)$ are chosen such that $\gamma_{i, n} \geq 0$ and

$$
\gamma_{i, n} \geq \max \left\{-\frac{\partial f}{\partial u}\left(x_{i}, u, v\right): \underline{u}_{i, n} \leq u \leq \bar{u}_{i, n} \text { and } \underline{u}_{i, n-s} \leq v \leq \bar{u}_{i, n-s}\right\} .
$$

A novelty of Theorem 2.1 is that no constraint condition is imposed on the time increment $k$ and $\mathbf{f}$ is only required to satisfy the local Lipschitz condition (2.4). Moreover, its proof is new and can not be contained in [12]. (b) To compute the solution $\mathbf{u}_{n}^{*}$, the monotone iteration processes given in [12] can be applied if the time increment $k$ satisfies constraint condition (2.11) in which $C^{1}$-smoothness of $\mathbf{f}$ is required. Indeed, the condition (2.11) is required in [12] to ensure the convergence of the iteration process to the solution $\mathbf{u}_{n}^{*}$.

Since the main concern of this paper is the asymptotic behavior of the time-dependent solution, the detailed discussion of the algorithm for the computation of the solution is omitted.

\section{Asymptotic Behavior of time-DePendent solutions}

Firstly, we introduce a pair of coupled upper and lower solutions of the system (1.4) as follows:

Definition 3.1. Two vectors $\overline{\mathbf{u}}, \underline{\mathbf{u}} \in \mathbb{R}^{N}$ are called a pair of coupled upper and lower solutions of the system (1.4) if

(i) $\overline{\mathbf{u}} \geq \underline{\mathbf{u}}$;

(ii) there exists a nonnegative diagonal matrix $C$ such that for all $\mathbf{w} \in \mathbf{K}(\underline{\mathbf{u}}, \overline{\mathbf{u}})$,

$$
\mathbf{f}(\mathbf{w}, \mathbf{u})-\mathbf{f}(\mathbf{w}, \mathbf{v}) \geq-C(\mathbf{u}-\mathbf{v})
$$

whenever $\underline{\mathbf{u}} \leq \mathbf{v} \leq \mathbf{u} \leq \overline{\mathbf{u}}$

(iii) for the nonnegative diagonal matrix $C$ in (3.1),

$$
\left\{\begin{array}{l}
A \overline{\mathbf{u}} \geq \mathbf{f}(\overline{\mathbf{u}}, \overline{\mathbf{u}})+C(\overline{\mathbf{u}}-\underline{\mathbf{u}}), \\
A \underline{\mathbf{u}} \leq \mathbf{f}(\underline{\mathbf{u}}, \underline{\mathbf{u}})-C(\overline{\mathbf{u}}-\underline{\mathbf{u}}) .
\end{array}\right.
$$

Remark 3.1. It is clear that if $\overline{\mathbf{u}}$ and $\underline{\mathbf{u}}$ are coupled upper and lower solutions of the system (1.4), the constant sequences $\overline{\mathbf{u}}_{n} \equiv \overline{\mathbf{u}}$ and $\underline{\mathbf{u}}_{n} \equiv \underline{\mathbf{u}}(n \geq-s)$ are also coupled upper and lower solutions of the system (1.3) whenever $\mathbf{\Psi}_{n} \in \mathbf{K}(\underline{\mathbf{u}}, \overline{\mathbf{u}})$ for $n=-s,-s+1, \ldots, 0$. This fact is crucial for our discussions.

Remark 3.2. At first glance, the construction of a pair of coupled upper and lower solutions seems to be complicated. But in fact this is not the case. It can always be easily implemented in specific problems. Such examples will be given in Section 4 .

Given two initial iterations $\overline{\mathbf{u}}^{(0)}$ and $\underline{\mathbf{u}}^{(0)}$, we consider the following iterative scheme:

$$
\left\{\begin{array}{l}
\left(A+\Gamma^{*}\right) \overline{\mathbf{u}}^{(m)}=\Gamma^{*} \overline{\mathbf{u}}^{(m-1)}+\mathbf{f}\left(\overline{\mathbf{u}}^{(m-1)}, \overline{\mathbf{u}}^{(m-1)}\right)+C^{*}\left(\overline{\mathbf{u}}^{(m-1)}-\underline{\mathbf{u}}^{(m-1)}\right), \\
\left(A+\Gamma^{*}\right) \underline{\mathbf{u}}^{(m)}=\Gamma^{*} \underline{\mathbf{u}}^{(m-1)}+\mathbf{f}\left(\underline{\mathbf{u}}^{(m-1)}, \underline{\mathbf{u}}^{(m-1)}\right)-C^{*}\left(\overline{\mathbf{u}}^{(m-1)}-\underline{\mathbf{u}}^{(m-1)}\right)
\end{array}\right.
$$

where $\Gamma^{*}$ and $C^{*}$ denote two nonnegative diagonal matrices specified later. It is clear that the sequences $\left\{\overline{\mathbf{u}}^{(m)}\right\}$ and $\left\{\underline{\mathbf{u}}^{(m)}\right\}$ given by the above iterative scheme is well defined provided that $\Gamma^{*} \neq 0$.

Theorem 3.1. Let $\overline{\mathbf{u}}$ and $\underline{\mathbf{u}}$ be a pair of coupled upper and lower solutions of the system (1.4), and $C$ be the nonnegative diagonal matrix in (3.1). Assume that there exists a nonnegative but non-zero diagonal matrix $\Gamma$ such that for all $\mathbf{w} \in \mathbf{K}(\underline{\mathbf{u}}, \overline{\mathbf{u}})$,

$$
\mathbf{f}(\mathbf{u}, \mathbf{w})-\mathbf{f}(\mathbf{v}, \mathbf{w}) \geq-\Gamma(\mathbf{u}-\mathbf{v})
$$


whenever $\underline{\mathbf{u}} \leq \mathbf{v} \leq \mathbf{u} \leq \overline{\mathbf{u}}$. Then the sequences $\left\{\overline{\mathbf{u}}^{(m)}\right\}$ and $\left\{\underline{\mathbf{u}}^{(m)}\right\}$ defined by the iterative scheme (3.3) with $\Gamma^{*}=\Gamma, C^{*}=C$ and the initial iterations $\overline{\mathbf{u}}^{(0)}=\overline{\mathbf{u}}$ and $\underline{\mathbf{u}}^{(0)}=\underline{\mathbf{u}}$ converge monotonically to their respective limits $\overline{\mathbf{u}}^{*}$ and $\underline{\mathbf{u}}^{*}$. Moreover for all $m \geq 1$,

$$
\underline{\mathbf{u}} \leq \underline{\mathbf{u}}^{(m)} \leq \underline{\mathbf{u}}^{(m+1)} \leq \underline{\mathbf{u}}^{*} \leq \overline{\mathbf{u}}^{*} \leq \overline{\mathbf{u}}^{(m+1)} \leq \overline{\mathbf{u}}^{(m)} \leq \overline{\mathbf{u}} .
$$

In addition, for any solution $\mathbf{u}$ of the system (1.4) in $\mathbf{K}(\underline{\mathbf{u}}, \overline{\mathbf{u}})$ we have $\mathbf{u} \in \mathbf{K}\left(\underline{\mathbf{u}}^{*}, \overline{\mathbf{u}}^{*}\right)$.

Proof. We firstly prove that for all $m \geq 1$,

$$
\underline{\mathbf{u}}^{(m-1)} \leq \underline{\mathbf{u}}^{(m)} \leq \overline{\mathbf{u}}^{(m)} \leq \overline{\mathbf{u}}^{(m-1)} .
$$

Let $\overline{\mathbf{w}}^{(0)}=\overline{\mathbf{u}}^{(0)}-\overline{\mathbf{u}}^{(1)}=\overline{\mathbf{u}}-\overline{\mathbf{u}}^{(1)}$. Then by (3.2) and (3.3) with $m=1,(A+\Gamma) \overline{\mathbf{w}}^{(0)} \geq \mathbf{0}$. Since $(A+\Gamma)^{-1} \geq 0$, we have $\overline{\mathbf{w}}^{(0)} \geq \mathbf{0}$ which gives $\overline{\mathbf{u}}^{(0)} \geq \overline{\mathbf{u}}^{(1)}$. Similarly, $\underline{\mathbf{u}}^{(1)} \geq \underline{\mathbf{u}}^{(0)}$. By (3.3), (3.1) and (3.4),

$$
(A+\Gamma)\left(\overline{\mathbf{u}}^{(1)}-\underline{\mathbf{u}}^{(1)}\right) \geq C\left(\overline{\mathbf{u}}^{(0)}-\underline{\mathbf{u}}^{(0)}\right)=C(\overline{\mathbf{u}}-\underline{\mathbf{u}}) \geq 0 .
$$

This proves $\overline{\mathbf{u}}^{(1)} \geq \underline{\mathbf{u}}^{(1)}$. The above conclusions show that

$$
\underline{\mathbf{u}}^{(0)} \leq \underline{\mathbf{u}}^{(1)} \leq \overline{\mathbf{u}}^{(1)} \leq \overline{\mathbf{u}}^{(0)} .
$$

The monotonicity property (3.6) follows from an inductive argument.

In view of the monotonicity property (3.6), there exist the limits

$$
\lim _{m \rightarrow \infty} \overline{\mathbf{u}}^{(m)}=\overline{\mathbf{u}}^{*}, \quad \lim _{m \rightarrow \infty} \underline{\mathbf{u}}^{(m)}=\underline{\mathbf{u}}^{*} .
$$

Moreover, (3.5) is valid.

Let $\mathbf{u}$ be any solution of the system (1.4) in $\mathbf{K}(\underline{\mathbf{u}}, \overline{\mathbf{u}})$. Suppose that $\mathbf{u} \in \mathbf{K}\left(\underline{\mathbf{u}}^{(m)}, \overline{\mathbf{u}}^{(m)}\right)$ for some $m \geq 0$. Then by (1.4), (3.3), (3.1) and (3.4),

$$
\begin{aligned}
(A+\Gamma)\left(\overline{\mathbf{u}}^{(m+1)}-\mathbf{u}\right) & =\Gamma\left(\overline{\mathbf{u}}^{(m)}-\mathbf{u}\right)+\mathbf{f}\left(\overline{\mathbf{u}}^{(m)}, \overline{\mathbf{u}}^{(m)}\right)-\mathbf{f}(\mathbf{u}, \mathbf{u})+C\left(\overline{\mathbf{u}}^{(m)}-\underline{\mathbf{u}}^{(m)}\right) \\
& \geq C\left(\mathbf{u}-\underline{\mathbf{u}}^{(m)}\right) \geq \mathbf{0} .
\end{aligned}
$$

This implies $\overline{\mathbf{u}}^{(m+1)} \geq \mathbf{u}$. Similarly, $\mathbf{u} \geq \underline{\mathbf{u}}^{(m+1)}$. It follows from the induction principle that $\mathbf{u} \in \mathbf{K}\left(\underline{\mathbf{u}}^{(m)}, \overline{\mathbf{u}}^{(m)}\right)$ for all $m \geq 0$. Letting $m \rightarrow \infty$, we get that $\mathbf{u} \in \mathbf{K}\left(\underline{\mathbf{u}}^{*}, \overline{\mathbf{u}}^{*}\right)$. This completes the proof.

Clearly, the limits $\overline{\mathbf{u}}^{*}$ and $\underline{\mathbf{u}}^{*}$ in Theorem 3.1 satisfy the relation

$$
\left\{\begin{array}{l}
A \overline{\mathbf{u}}^{*}=\mathbf{f}\left(\overline{\mathbf{u}}^{*}, \overline{\mathbf{u}}^{*}\right)+C\left(\overline{\mathbf{u}}^{*}-\underline{\mathbf{u}}^{*}\right), \\
A \underline{\mathbf{u}}^{*}=\mathbf{f}\left(\underline{\mathbf{u}}^{*}, \underline{\mathbf{u}}^{*}\right)-C\left(\overline{\mathbf{u}}^{*}-\underline{\mathbf{u}}^{*}\right) .
\end{array}\right.
$$

We call $\overline{\mathbf{u}}^{*}$ and $\underline{\mathbf{u}}^{*}$ the coupled quasi-solutions of the system (1.4). In general, they are not true solutions. However, if $\mathbf{f}(\mathbf{u}, \mathbf{v})$ is monotone nondecreasing in $\mathbf{v}$ for all $\mathbf{u}, \mathbf{v} \in \mathbf{K}(\underline{\mathbf{u}}, \overline{\mathbf{u}})$, we take $C=0$ in (3.1) and so $\overline{\mathbf{u}}^{*}$ and $\underline{\mathbf{u}}^{*}$ are the maximal and minimal solutions of the system (1.4) in $\mathbf{K}(\underline{\mathbf{u}}, \overline{\mathbf{u}})$, respectively. In the general case, if $\overline{\mathbf{u}}^{*}=\underline{\mathbf{u}}^{*}$ then $\overline{\mathbf{u}}^{*}$ (or $\underline{\mathbf{u}}^{*}$ ) is the unique solution of the system (1.4) in $\mathbf{K}(\underline{\mathbf{u}}, \overline{\mathbf{u}})$.

Let $\rho(\cdot)$ denote the spectral radius of the corresponding matrix. Hypothesis $(\mathcal{H})$ ensures that for each real number $\sigma>0,(A+\sigma I)^{-1} \geq 0$ and so $\rho\left((A+\sigma I)^{-1}\right)$ is a real eigenvalue of $(A+\sigma I)^{-1}$ (see [3,17]). This property 
implies that $A$ has at least one real eigenvalue and its smallest real eigenvalue, denoted by $\lambda_{0}$, is nonnegative. In fact, $\lambda_{0}$ is positive if the strict inequality $\sum_{j=1}^{N} a_{i, j}>0$ holds for at least one $i$. In terms of $\lambda_{0}$, the following theorem gives a sufficient condition ensuring $\overline{\mathbf{u}}^{*}=\underline{\mathbf{u}}^{*}$.

Theorem 3.2. Let the conditions in Theorem 3.1 hold. In addition, assume that there exists a diagonal matrix $\Gamma^{\prime}$ such that

$$
\mathbf{f}(\mathbf{u}, \mathbf{u})-\mathbf{f}(\mathbf{v}, \mathbf{v}) \leq \Gamma^{\prime}(\mathbf{u}-\mathbf{v})
$$

whenever $\underline{\mathbf{u}} \leq \mathbf{v} \leq \mathbf{u} \leq \overline{\mathbf{u}}$. If $\Gamma^{\prime}+2 C<\lambda_{0}$ I, then $\overline{\mathbf{u}}^{*}=\underline{\mathbf{u}}^{*}$ and $\overline{\mathbf{u}}^{*}$ (or $\underline{\mathbf{u}}^{*}$ ) is the unique solution of the system (1.4) in $\mathbf{K}(\underline{\mathbf{u}}, \overline{\mathbf{u}})$.

Proof. Since $\underline{\mathbf{u}} \leq \underline{\mathbf{u}}^{*} \leq \overline{\mathbf{u}}^{*} \leq \overline{\mathbf{u}}$, it suffices to show $\overline{\mathbf{u}}^{*} \leq \underline{\mathbf{u}}^{*}$. Clearly,

$$
\begin{aligned}
A\left(\overline{\mathbf{u}}^{*}-\underline{\mathbf{u}}^{*}\right) & =\mathbf{f}\left(\overline{\mathbf{u}}^{*}, \overline{\mathbf{u}}^{*}\right)-\mathbf{f}\left(\underline{\mathbf{u}}^{*}, \underline{\mathbf{u}}^{*}\right)+2 C\left(\overline{\mathbf{u}}^{*}-\underline{\mathbf{u}}^{*}\right) \\
& \leq\left(\Gamma^{\prime}+2 C\right)\left(\overline{\mathbf{u}}^{*}-\underline{\mathbf{u}}^{*}\right)
\end{aligned}
$$

or equivalently,

$$
\left(A-\Gamma^{\prime}-2 C\right)\left(\overline{\mathbf{u}}^{*}-\underline{\mathbf{u}}^{*}\right) \leq \mathbf{0} .
$$

Let $\Gamma^{\prime}+2 C=\operatorname{diag}\left(\sigma_{1}, \sigma_{2}, \ldots, \sigma_{N}\right)$. Set $\bar{\sigma}=\max _{1 \leq i \leq N} \sigma_{i}$ and $\underline{\sigma}=\min _{1 \leq i \leq N} \sigma_{i}$. Since $\Gamma^{\prime}+2 C<\lambda_{0} I$, we have that $\bar{\sigma}<\lambda_{0}$ and $\underline{\sigma}<\lambda_{0}$. Taking $\delta<\min (0, \underline{\sigma})$ and defining $\bar{A}=A-\delta I$, we have that $\bar{A}^{-1} \geq 0$ and $0<(\underline{\sigma}-\delta) I \leq \Gamma^{\prime}+2 C-\delta I \leq(\bar{\sigma}-\delta) I$. Next we write

$$
A-\Gamma^{\prime}-2 C=\bar{A}\left(I-\bar{A}^{-1}\left(\Gamma^{\prime}+2 C-\delta I\right)\right) .
$$

Since

$$
0 \leq \bar{A}^{-1}\left(\Gamma^{\prime}+2 C-\delta I\right) \leq(\bar{\sigma}-\delta) \bar{A}^{-1}
$$

we have $\left.\rho\left(\bar{A}^{-1} \Gamma^{\prime}+2 C-\delta I\right)\right) \leq(\bar{\sigma}-\delta) \rho\left(\bar{A}^{-1}\right)$ (see [9]). On the other hand, the non-negativity of $\bar{A}^{-1}$ implies that there exists a real eigenvalue $\lambda$ of $A$ such that $\rho\left(\bar{A}^{-1}\right)=(\lambda-\delta)^{-1}$. Hence,

$$
\rho\left(\bar{A}^{-1}\left(\Gamma^{\prime}+2 C-\delta I\right)\right) \leq \frac{\bar{\sigma}-\delta}{\lambda-\delta} \leq \frac{\bar{\sigma}-\delta}{\lambda_{0}-\delta}<1
$$

This proves that $\left(I-\bar{A}^{-1}\left(\Gamma^{\prime}+2 C-\delta I\right)\right)^{-1}$ exists and is nonnegative (see [9]). By (3.10), we obtain that $\left(A-\Gamma^{\prime}-2 C\right)^{-1}$ exists and is nonnegative. It follows from (3.9) that $\overline{\mathbf{u}}^{*} \leq \underline{\mathbf{u}}^{*}$. This completes the proof.

Remark 3.3. If $\mathbf{f}(\mathbf{u}, \mathbf{v})$ is monotone nondecreasing in $\mathbf{v}$ for all $\mathbf{u}, \mathbf{v} \in \mathbf{K}(\underline{\mathbf{u}}, \overline{\mathbf{u}})$, we take $C=0$ in (3.1) and so the condition $\Gamma^{\prime}+2 C<\lambda_{0} I$ is reduced to one of Theorem 2.3 in [11].

Let $\overline{\mathbf{u}}$ and $\underline{\mathbf{u}}$ be a pair of coupled upper and lower solutions of the system (1.4), and let the local Lipschitz condition (3.4) hold. Also let $\overline{\mathbf{u}}^{*}$ and $\underline{\mathbf{u}}^{*}$ be the limits in Theorem 3.1. When $\mathbf{\Psi}_{n} \in \mathbf{K}(\underline{\mathbf{u}}, \overline{\mathbf{u}})$ for $n=-s,-s+$ $1, \ldots, 0$, we have from Theorem 2.1 that there is at least one solution of the system (1.3) in $\mathbf{K}(\underline{\mathbf{u}}, \overline{\mathbf{u}})$ since the constant sequences $\overline{\mathbf{u}}_{n} \equiv \overline{\mathbf{u}}$ and $\underline{\mathbf{u}}_{n} \equiv \underline{\mathbf{u}}(n \geq-s)$ are also the coupled upper and lower solutions of the system (1.3). Next, we show that the sector $\mathbf{K}\left(\underline{\mathbf{u}}^{*}, \overline{\mathbf{u}}^{*}\right)$ is an attractor of the solution of the system (1.3) in $\mathbf{K}(\underline{\mathbf{u}}, \overline{\mathbf{u}})$. 
Theorem 3.3. Let $\overline{\mathbf{u}}$ and $\underline{\mathbf{u}}$ be a pair of coupled upper and lower solutions of the system (1.4), and let the local Lipschitz condition (3.4) hold. Also let $\overline{\mathbf{u}}^{*}$ and $\underline{\mathbf{u}}^{*}$ be the limits in Theorem 3.1. Then when $\mathbf{\Psi}_{n} \in \mathbf{K}(\underline{\mathbf{u}}, \overline{\mathbf{u}})$ for $n=-s,-s+1, \ldots, 0$, any solution $\mathbf{u}_{n}^{*}$ of the system (1.3) in $\mathbf{K}(\underline{\mathbf{u}}, \overline{\mathbf{u}})$ satisfies the relation

$$
\underline{\mathbf{u}}^{*} \leq \mathbf{u}_{n}^{*} \leq \overline{\mathbf{u}}^{*}, \quad \text { as } n \rightarrow \infty .
$$

Proof. Let $C$ be the nonnegative diagonal matrix in (3.1). We embed the system (1.3) into the following extended system:

$$
\left\{\begin{array}{c}
(I+k A) \mathbf{u}_{n}=\mathbf{u}_{n-1}+k\left(\mathbf{f}\left(\mathbf{u}_{n}, \mathbf{u}_{n-s}\right)+C\left(\mathbf{u}_{n-s}+\mathbf{v}_{n-s}\right)\right) \\
(I+k A) \mathbf{v}_{n}=\mathbf{v}_{n-1}+k\left(-\mathbf{f}\left(-\mathbf{v}_{n},-\mathbf{v}_{n-s}\right)+C\left(\mathbf{u}_{n-s}+\mathbf{v}_{n-s}\right)\right), \\
n=1,2, \ldots, \\
\mathbf{u}_{n}=\mathbf{\Psi}_{n}, \mathbf{v}_{n}=\mathbf{\Phi}_{n}, \quad n=-s,-s+1, \ldots, 0
\end{array}\right.
$$

Clearly, if $\mathbf{u}_{n}^{*}$ is a solution of (1.3) then $\left(\mathbf{u}_{n}, \mathbf{v}_{n}\right)=\left(\mathbf{u}_{n}^{*},-\mathbf{u}_{n}^{*}\right)$ is a solution of (3.13) provided $\boldsymbol{\Phi}_{n}=-\boldsymbol{\Psi}_{n}$. The corresponding "steady-state" problem of (3.13) is given by

$$
\left\{\begin{array}{l}
A \mathbf{u}=\mathbf{f}(\mathbf{u}, \mathbf{u})+C(\mathbf{u}+\mathbf{v}) \\
A \mathbf{v}=-\mathbf{f}(-\mathbf{v},-\mathbf{v})+C(\mathbf{u}+\mathbf{v})
\end{array}\right.
$$

Since $C$ is a nonnegative diagonal matrix, the right-hand side of the above problem (3.14) is a quasi-monotone nondecreasing function (see [15] for the definition of a quasi-monotone nondecreasing function). Define

$$
\begin{aligned}
& \overline{\mathbf{w}}=(\overline{\mathbf{u}},-\underline{\mathbf{u}}), \quad \underline{\mathbf{w}}=(\underline{\mathbf{u}},-\overline{\mathbf{u}}), \\
& \overline{\mathbf{w}}^{*}=\left(\overline{\mathbf{u}}^{*},-\underline{\mathbf{u}}^{*}\right), \underline{\mathbf{w}}^{*}=\left(\underline{\mathbf{u}}^{*},-\overline{\mathbf{u}}^{*}\right) .
\end{aligned}
$$

Let $\overline{\mathbf{u}}^{(m)}$ and $\underline{\mathbf{u}}^{(m)}$ be the sequences given by (3.3) with $\Gamma^{*}=\Gamma, C^{*}=C$ and the initial iterations $\overline{\mathbf{u}}^{(0)}=\overline{\mathbf{u}}$ and $\underline{\mathbf{u}}^{(0)}=\underline{\mathbf{u}}$, where $\Gamma$ is the nonnegative matrix in (3.4). Then by Theorem 3.1,

$$
\lim _{m \rightarrow \infty}\left(\overline{\mathbf{u}}^{(m)}, \overline{\mathbf{v}}^{(m)}\right)=\overline{\mathbf{w}}^{*}
$$

where $\overline{\mathbf{v}}^{(m)}=-\underline{\mathbf{u}}^{(m)}$. Moreover by (3.3), the sequence $\left\{\left(\overline{\mathbf{u}}^{(m)}, \overline{\mathbf{v}}^{(m)}\right)\right\}$ is governed by the following iteration process

$$
\left\{\begin{array}{l}
(A+\Gamma) \overline{\mathbf{u}}^{(m)}=\Gamma \overline{\mathbf{u}}^{(m-1)}+\mathbf{f}\left(\overline{\mathbf{u}}^{(m-1)}, \overline{\mathbf{u}}^{(m-1)}\right)+C\left(\overline{\mathbf{u}}^{(m-1)}+\overline{\mathbf{v}}^{(m-1)}\right), \\
(A+\Gamma) \overline{\mathbf{v}}^{(m)}=\Gamma \overline{\mathbf{v}}^{(m-1)}-\mathbf{f}\left(-\overline{\mathbf{v}}^{(m-1)},-\overline{\mathbf{v}}^{(m-1)}\right)+C\left(\overline{\mathbf{u}}^{(m-1)}+\overline{\mathbf{v}}^{(m-1)}\right)
\end{array}\right.
$$

with the initial iteration $\left(\overline{\mathbf{u}}^{(0)}, \overline{\mathbf{v}}^{(0)}\right)=\overline{\mathbf{w}}$. Since $\overline{\mathbf{u}}$ and $\underline{\mathbf{u}}$ are the coupled upper and lower solutions of (1.4), it is easy to verify from (3.2) that $\overline{\mathbf{w}}$ and $\underline{\mathbf{w}}$ are ordered upper and lower solutions of (3.14) under the usual definition (see [15]). Using the usual method of upper and lower solutions as that in [15] we obtain that the limit $\overline{\mathbf{w}}^{*}$ is the maximal solution of $(3.14)$ in $\mathbf{K}(\underline{\mathbf{w}}, \overline{\mathbf{w}})$. Similarly, $\underline{\mathbf{w}}^{*}$ is the minimal solution of $(3.14)$ in $\mathbf{K}(\underline{\mathbf{w}}, \overline{\mathbf{w}})$.

Define

$$
\mathbf{g}_{1}(\mathbf{u}, \mathbf{v})=\mathbf{f}(\mathbf{u}, \mathbf{v})+\Gamma \mathbf{u}+C \mathbf{v}, \quad \mathbf{g}_{2}(\mathbf{u}, \mathbf{v})=-\mathbf{g}_{1}(-\mathbf{u},-\mathbf{v})
$$


It follows from (3.1) and (3.4) that $\mathbf{g}_{1}(\mathbf{u}, \mathbf{v})$ is monotone nondecreasing for all $\mathbf{u}, \mathbf{v} \in \mathbf{K}(\underline{\mathbf{u}}, \overline{\mathbf{u}})$ and $\mathbf{g}_{2}(\mathbf{u}, \mathbf{v})$ is monotone nondecreasing for all $\mathbf{u}, \mathbf{v} \in \mathbf{K}(-\overline{\mathbf{u}},-\underline{\mathbf{u}})$. Moreover by the usual definition, the constant sequences $\overline{\mathbf{w}}_{n} \equiv \overline{\mathbf{w}}$ and $\underline{\mathbf{w}}_{n} \equiv \underline{\mathbf{w}}(n \geq-s)$ are ordered upper and lower solutions of (3.13) when $\left(\Psi_{n}, \mathbf{\Phi}_{n}\right) \in \mathbf{K}(\underline{\mathbf{w}}, \overline{\mathbf{w}})$ for $n=-s,-s+1, \ldots, 0$. Therefore, using the usual method of upper and lower solutions as that in [13] we obtain the existence of the solution of (3.13) in $\mathbf{K}(\underline{\mathbf{w}}, \overline{\mathbf{w}})$ for arbitrary initial function $\left(\mathbf{\Psi}_{n}, \boldsymbol{\Phi}_{n}\right) \in \mathbf{K}(\underline{\mathbf{w}}, \overline{\mathbf{w}})$. In particular, let $\left\{\overline{\mathbf{w}}_{n}^{(m)}\right\}=\left\{\left(\overline{\mathbf{u}}_{n}^{(m)}, \overline{\mathbf{v}}_{n}^{(m)}\right)\right\}$ and $\left\{\underline{\mathbf{w}}_{n}^{(m)}\right\}=\left\{\left(\underline{\mathbf{u}}_{n}^{(m)}, \underline{\mathbf{v}}_{n}^{(m)}\right)\right\}$ be the sequences governed by the iteration processes

$$
\left\{\begin{array}{c}
(I+k A+k \Gamma) \overline{\mathbf{u}}_{n}^{(m)}=\overline{\mathbf{u}}_{n-1}^{(m)}+k\left(\Gamma \overline{\mathbf{u}}_{n}^{(m-1)}+\mathbf{f}\left(\overline{\mathbf{u}}_{n}^{(m-1)}, \overline{\mathbf{u}}_{n-s}^{(m)}\right)+C\left(\overline{\mathbf{u}}_{n-s}^{(m)}+\overline{\mathbf{v}}_{n-s}^{(m)}\right)\right), \\
(I+k A+k \Gamma) \overline{\mathbf{v}}_{n}^{(m)}=\overline{\mathbf{v}}_{n-1}^{(m)}+k\left(\Gamma \overline{\mathbf{v}}_{n}^{(m-1)}-\mathbf{f}\left(-\overline{\mathbf{v}}_{n}^{(m-1)},-\overline{\mathbf{v}}_{n-s}^{(m)}\right)+C\left(\overline{\mathbf{u}}_{n-s}^{(m)}+\overline{\mathbf{v}}_{n-s}^{(m)}\right)\right), \\
n=1,2, \ldots, \\
\overline{\mathbf{u}}_{n}^{(m)}=\overline{\mathbf{u}}, \quad \overline{\mathbf{v}}_{n}^{(m)}=-\underline{\mathbf{u}}, \quad n=-s,-s+1, \ldots, 0
\end{array}\right.
$$

and

$$
\left\{\begin{array}{c}
(I+k A+k \Gamma) \underline{\mathbf{u}}_{n}^{(m)}=\underline{\mathbf{u}}_{n-1}^{(m)}+k\left(\Gamma \underline{\mathbf{u}}_{n}^{(m-1)}+\mathbf{f}\left(\underline{\mathbf{u}}_{n}^{(m-1)}, \underline{\mathbf{u}}_{n-s}^{(m)}\right)+C\left(\underline{\mathbf{u}}_{n-s}^{(m)}+\underline{\mathbf{v}}_{n-s}^{(m)}\right)\right), \\
(I+k A+k \Gamma) \underline{\mathbf{v}}_{n}^{(m)}=\underline{\mathbf{v}}_{n-1}^{(m)}+k\left(\Gamma \underline{\mathbf{v}}_{n}^{(m-1)}-\mathbf{f}\left(-\underline{\mathbf{v}}_{n}^{(m-1)},-\underline{\mathbf{v}}_{n-s}^{(m)}\right)+C\left(\underline{\mathbf{u}}_{n-s}^{(m)}+\underline{\mathbf{v}}_{n-s}^{(m)}\right)\right), \\
n=1,2, \ldots, \\
\underline{\mathbf{u}}_{n}^{(m)}=\underline{\mathbf{u}}, \quad \underline{\mathbf{v}}_{n}^{(m)}=-\overline{\mathbf{u}}, \quad n=-s,-s+1, \ldots, 0
\end{array}\right.
$$

with the initial iterations $\overline{\mathbf{w}}_{n}^{(0)}=\overline{\mathbf{w}}$ and $\underline{\mathbf{w}}_{n}^{(0)}=\underline{\mathbf{w}}$ for $n=-s,-s+1, \ldots$ Then there exist the limits

$$
\lim _{m \rightarrow \infty} \overline{\mathbf{w}}_{n}^{(m)}=\overline{\mathbf{w}}_{n}^{*}, \quad \lim _{m \rightarrow \infty} \underline{\mathbf{w}}_{n}^{(m)}=\underline{\mathbf{w}}_{n}^{*}
$$

and the limits $\overline{\mathbf{w}}_{n}^{*}, \underline{\mathbf{w}}_{n}^{*} \in \mathbf{K}(\underline{\mathbf{w}}, \overline{\mathbf{w}})$. Moreover, $\overline{\mathbf{w}}_{n}^{*}$ is the solution of the problem (3.13) with $\left(\mathbf{\Psi}_{n}, \mathbf{\Phi}_{n}\right)=\overline{\mathbf{w}}$, while $\underline{\mathbf{w}}_{n}^{*}$ is the solution of the problem (3.13) with $\left(\boldsymbol{\Psi}_{n}, \mathbf{\Phi}_{n}\right)=\underline{\mathbf{w}}$.

Let $\mathbf{w}_{n}^{*}$ be the solution of (3.13) in $\mathbf{K}(\underline{\mathbf{w}}, \overline{\mathbf{w}})$ for arbitrary initial function $\left(\mathbf{\Psi}_{n}, \mathbf{\Phi}_{n}\right) \in \mathbf{K}(\underline{\mathbf{w}}, \overline{\mathbf{w}})$. Also let $\mathbf{w}^{*}$ be any solution of (3.14) in $\mathbf{K}(\underline{\mathbf{w}}, \overline{\mathbf{w}})$. Then an inductive argument using $(3.16,3.17)$, the nonnegative property $(I+k A+k \Gamma)^{-1}$ and the monotone nondecreasing property of the functions $\mathbf{g}_{1}$ and $\mathbf{g}_{2}$ gives that for all $m \geq 0$ and $n=-s,-s+1, \ldots$,

$$
\begin{gathered}
\overline{\mathbf{w}}_{n}^{(m)} \leq \overline{\mathbf{w}}_{n-1}^{(m)}, \quad \underline{\mathbf{w}}_{n-1}^{(m)} \leq \underline{\mathbf{w}}_{n}^{(m)}, \\
\underline{\mathbf{w}}_{n}^{(m)} \leq \mathbf{w}^{*} \leq \overline{\mathbf{w}}_{n}^{(m)}, \quad \underline{\mathbf{w}}_{n}^{(m)} \leq \mathbf{w}_{n}^{*} \leq \overline{\mathbf{w}}_{n}^{(m)} .
\end{gathered}
$$

The above relations imply that the sequence $\left\{\overline{\mathbf{w}}_{n}^{*}\right\}$ converges monotonically from above to the maximal solution $\overline{\mathbf{w}}^{*}$ of (3.14) in $\mathbf{K}(\underline{\mathbf{w}}, \overline{\mathbf{w}})$ as $n \rightarrow \infty$, and the sequence $\left\{\underline{\mathbf{w}}_{n}^{*}\right\}$ converges monotonically from below to the minimal solution $\underline{\mathbf{w}}^{*}$ of $(3.14)$ in $\mathbf{K}(\underline{\mathbf{w}}, \overline{\mathbf{w}})$. Moreover, for arbitrary initial function $\left(\boldsymbol{\Psi}_{n}, \boldsymbol{\Phi}_{n}\right) \in \mathbf{K}(\underline{\mathbf{w}}, \overline{\mathbf{w}})$ the corresponding solution $\mathbf{w}_{n}^{*}$ of $(3.13)$ in $\mathbf{K}(\underline{\mathbf{w}}, \overline{\mathbf{w}})$ satisfies the relation

$$
\underline{\mathbf{w}}^{*} \leq \mathbf{w}_{n}^{*} \leq \overline{\mathbf{w}}^{*}, \quad \text { as } n \rightarrow \infty .
$$

Let $\mathbf{u}_{n}^{*}$ be the solution of (1.3) in $\mathbf{K}(\underline{\mathbf{u}}, \overline{\mathbf{u}})$ with the initial function $\mathbf{\Psi}_{n} \in \mathbf{K}(\underline{\mathbf{u}}, \overline{\mathbf{u}})$. Set $\mathbf{w}_{n}=\left(\mathbf{u}_{n}^{*},-\mathbf{u}_{n}^{*}\right)$. Then $\mathbf{w}_{n} \in \mathbf{K}(\underline{\mathbf{w}}, \overline{\mathbf{w}})$ and it is a solution of (3.13) with $\mathbf{\Phi}_{n}=-\mathbf{\Psi}_{n}$. So by (3.18),

$$
\underline{\mathbf{w}}^{*} \leq \mathbf{w}_{n} \leq \overline{\mathbf{w}}^{*}, \quad \text { as } n \rightarrow \infty
$$


which leads to

$$
\underline{\mathbf{u}}^{*} \leq \mathbf{u}_{n}^{*} \leq \overline{\mathbf{u}}^{*}, \quad \text { as } n \rightarrow \infty
$$

This proves the theorem.

The relation (3.12) implies that the sector $\mathbf{K}\left(\underline{\mathbf{u}}^{*}, \overline{\mathbf{u}}^{*}\right)$ between the two coupled quasi-solutions $\underline{\mathbf{u}}^{*}$ and $\overline{\mathbf{u}}^{*}$ of (1.4) is an attractor of the solution $\mathbf{u}_{n}^{*}$ of (1.3) in $\mathbf{K}(\underline{\mathbf{u}}, \overline{\mathbf{u}})$.

As a consequence of Theorem 3.3 we have the following convergence result.

Corollary 3.1. Let the conditions in Theorem 3.3 be satisfied. If $\underline{\mathbf{u}}^{*}=\overline{\mathbf{u}}^{*} \equiv \mathbf{u}^{*}$ then $\mathbf{u}^{*}$ is the unique solution of (1.4) in $\mathbf{K}(\underline{\mathbf{u}}, \overline{\mathbf{u}})$, and when $\mathbf{\Psi}_{n} \in \mathbf{K}(\underline{\mathbf{u}}, \overline{\mathbf{u}})$ for $n=-s,-s+1, \ldots, 0$, the corresponding solution $\mathbf{u}_{n}^{*}$ of (1.3) in $\mathbf{K}(\underline{\mathbf{u}}, \overline{\mathbf{u}})$ converges to $\mathbf{u}^{*}$ as $n \rightarrow \infty$.

A sufficient condition ensuring $\overline{\mathbf{u}}^{*}=\underline{\mathbf{u}}^{*}$ is given in Theorem 3.2. The following theorem ensures that $\overline{\mathbf{u}}^{*}=\underline{\mathbf{u}}^{*}$ if and only if the solution of the problem (3.14) is unique.

Theorem 3.4. Let the conditions in Theorem 3.3 be satisfied. Let $\overline{\mathbf{w}}=(\overline{\mathbf{u}},-\underline{\mathbf{u}})$ and $\underline{\mathbf{w}}=(\underline{\mathbf{u}},-\overline{\mathbf{u}})$. Then $\overline{\mathbf{u}}^{*}=\underline{\mathbf{u}}^{*}$ if and only if the solution of (3.14) is unique in $\mathbf{K}(\underline{\mathbf{w}}, \overline{\mathbf{w}})$.

Proof. By the proof of Theorem 3.3, the pair $\left(\overline{\mathbf{u}}^{*},-\underline{\mathbf{u}}^{*}\right)$ and $\left(\underline{\mathbf{u}}^{*},-\overline{\mathbf{u}}^{*}\right)$ are the maximal and minimal solutions of (3.14) in $\mathbf{K}(\underline{\mathbf{w}}, \overline{\mathbf{w}})$. Hence, if the solution of $(3.14)$ is unique in $\mathbf{K}(\underline{\mathbf{w}}, \overline{\mathbf{w}})$, then $\left(\overline{\mathbf{u}}^{*},-\underline{\mathbf{u}}^{*}\right)=\left(\underline{\mathbf{u}}^{*},-\overline{\mathbf{u}}^{*}\right)$ which implies $\overline{\mathbf{u}}^{*}=\underline{\mathbf{u}}^{*}$. Conversely, if $\overline{\mathbf{u}}^{*}=\underline{\mathbf{u}}^{*}$ then $\left(\overline{\mathbf{u}}^{*},-\underline{\mathbf{u}}^{*}\right)=\left(\underline{\mathbf{u}}^{*},-\overline{\mathbf{u}}^{*}\right)$. It follows from the maximal and minimal property of $\left(\overline{\mathbf{u}}^{*},-\underline{\mathbf{u}}^{*}\right)$ and $\left(\underline{\mathbf{u}}^{*},-\overline{\mathbf{u}}^{*}\right)$ that the problem $(3.14)$ has exactly one solution in $\mathbf{K}(\underline{\mathbf{w}}, \overline{\mathbf{w}})$.

\section{Applications and numerical Results}

As an application of the results given in Section 3 we consider the following reaction diffusion problem with time delay:

$$
\left\{\begin{array}{l}
\frac{\partial u}{\partial t}-D \frac{\partial^{2} u}{\partial x^{2}}=f(x, u(x, t), u(x, t-\tau)), \quad 0<x<1, t>0 \\
u(0, t)=u(1, t)=0, \quad t>0 \\
u(x, t)=\psi(x, t), \quad 0<x<1,-\tau \leq t \leq 0
\end{array}\right.
$$

where $D$ is a positive constant, and $\psi(x, t)$ is a continuous function in $(0,1) \times[-\tau, 0]$. For this problem, the finite difference approximation (1.3) reads as follows:

$$
\left\{\begin{array}{l}
\left(I+\frac{k D}{h^{2}} L\right) \mathbf{u}_{n}=\mathbf{u}_{n-1}+k \mathbf{f}\left(\mathbf{u}_{n}, \mathbf{u}_{n-s}\right), \quad n=1,2, \ldots \\
\mathbf{u}_{n}=\Psi_{n}, \quad n=-s,-s+1, \ldots, 0
\end{array}\right.
$$

where $h=1 /(N+1)$ and $L$ is an $N$ by $N$ tridiagonal matrix defined by

$$
L=\operatorname{tridiag}\{-1,2,-1\}
$$

Similarly, the finite difference approximation for the corresponding steady-state problem

$$
\left\{\begin{array}{l}
-D \frac{\partial^{2} u}{\partial x^{2}}=f(x, u, u), \quad 0<x<1 \\
u(0)=u(1)=0
\end{array}\right.
$$


is given by

$$
\frac{D}{h^{2}} L \mathbf{u}=\mathbf{f}(\mathbf{u}, \mathbf{u})
$$

In these problems, the functions $\mathbf{f}\left(\mathbf{u}_{n}, \mathbf{u}_{n-s}\right)$ and $\mathbf{f}(\mathbf{u}, \mathbf{u})$ are given as follows

$$
\begin{aligned}
& \mathbf{f}\left(\mathbf{u}_{n}, \mathbf{u}_{n-s}\right) \equiv\left(f\left(x_{1}, u_{1, n}, u_{1, n-s}\right), \ldots, f\left(x_{N}, u_{N, n}, u_{N, n-s}\right)\right)^{T} \\
& \mathbf{f}(\mathbf{u}, \mathbf{u}) \equiv\left(f\left(x_{1}, u_{1}, u_{1}\right), \ldots, f\left(x_{N}, u_{N}, u_{N}\right)\right)^{T}
\end{aligned}
$$

Clearly, the conditions in hypothesis $(\mathcal{H})$ are satisfied for this example.

\subsection{The construction of the coupled upper and lower solutions}

To apply the conclusions in Section 3 the main task is the construction of the coupled upper and lower solutions. It depends strongly on the function $f(x, u(x, t), u(x, t-\tau))$. Here, we discuss three special types of $f(x, u(x, t), u(x, t-\tau))$ each of which is not monotone in $u(x, t-\tau)$.

Example 1. The first example is for the function

$$
f(x, u(x, t), u(x, t-\tau))=u(x, t)(u(x, t-\tau)-\theta)(1-u(x, t-\tau))
$$

where $\theta$ is a positive constant. The problem $(4.1,4.5)$ describes the Fisher's model in population genetics (see $[2,13,14])$. It is easy to see that the function $f$ is neither nondecreasing nor nonincreasing in $u(x, t-\tau)$.

To find a pair of coupled upper and lower solutions of (4.4), we observe that the function $H(v) \equiv(v-\theta)(1-v)$ has a global maximal value $(1-\theta)^{2} / 4$ at $v=(1+\theta) / 2$.

Let $x_{i}=i h, i=0, \ldots, N+1$, be the mesh points, and define $\bar{u}_{i}=x_{i}\left(1-x_{i}\right)$ for all $i=0, \ldots, N+1$. We have $0 \leq \bar{u}_{i} \leq 1 / 4$ for all $i=0, \ldots, N+1$. Let $\overline{\mathbf{u}}=\left(\bar{u}_{1}, \bar{u}_{2}, \ldots, \bar{u}_{N}\right)^{T}$ and $\underline{\mathbf{u}}=\left(-\bar{u}_{1},-\bar{u}_{2}, \ldots,-\bar{u}_{N}\right)^{T}$. We have that for all $\mathbf{w} \in \mathbf{K}(\overline{\mathbf{u}}, \underline{\mathbf{u}})$,

$$
\mathbf{f}(\mathbf{w}, \mathbf{u})-\mathbf{f}(\mathbf{w}, \mathbf{v}) \geq-\left(\frac{3}{8}+\frac{\theta}{4}\right)(\mathbf{u}-\mathbf{v})
$$

whenever $\underline{\mathbf{u}} \leq \mathbf{v} \leq \mathbf{u} \leq \overline{\mathbf{u}}$. Also, we have that if

$$
D \geq \frac{(1-\theta)^{2}}{32}+\frac{1}{4}\left(\frac{3}{8}+\frac{\theta}{4}\right)
$$

then

$$
\left\{\begin{array}{l}
\frac{D}{h^{2}} L \overline{\mathbf{u}}=2 D \mathbf{z} \geq \mathbf{f}(\overline{\mathbf{u}}, \overline{\mathbf{u}})+\left(\frac{3}{8}+\frac{\theta}{4}\right)(\overline{\mathbf{u}}-\underline{\mathbf{u}}), \\
\frac{D}{h^{2}} L \underline{\mathbf{u}}=-2 D \mathbf{z} \leq \mathbf{f}(\underline{\mathbf{u}}, \underline{\mathbf{u}})-\left(\frac{3}{8}+\frac{\theta}{4}\right)(\overline{\mathbf{u}}-\underline{\mathbf{u}})
\end{array}\right.
$$

where $\mathbf{z}=(1,1, \ldots, 1)^{T} \in \mathbb{R}^{N}$. Take $C=(3 / 8+\theta / 4) I$. It follows from (4.8) that $\overline{\mathbf{u}}$ and $\underline{\mathbf{u}}$ are the coupled upper and lower solutions of (4.4) under the condition (4.7). Let $\overline{\mathbf{u}}_{n} \equiv \overline{\mathbf{u}}$ and $\underline{\mathbf{u}}_{n} \equiv \underline{\mathbf{u}}(n \geq-s)$. Then the constant sequences $\overline{\mathbf{u}}_{n}$ and $\underline{\mathbf{u}}_{n}$ are the coupled upper and lower solutions of (4.2) with $C_{n} \equiv C$ provided that the condition (4.7) holds and $-x(1-x) \leq \psi(x, t) \leq x(1-x)$ for $x \in(0,1)$ and $t \in[-\tau, 0]$. Hence the results in Section 3 can be applied to this example. 
Another pair of the coupled upper and lower solutions exists. Assume that $D>(1-\theta)^{2} / 32$. We choose a positive constant $\gamma$ such that

$$
D \geq \frac{(1-\theta)^{2}}{32}+\frac{\gamma^{2}}{128}+\frac{\gamma(1+\theta)}{32}
$$

and consider the linear equation

$$
\frac{1}{h^{2}} L \mathbf{u}=\gamma \mathbf{z}
$$

Clearly, there exists a unique positive solution $\widetilde{\mathbf{u}}$ to the above equation. Let $\tilde{l}_{i, j}$ be the elements of the matrix $L^{-1}$. Then

$$
\tilde{l}_{i, j}= \begin{cases}\frac{(N+1-j) i}{N+1}, & i \leq j, \\ \frac{(N+1-i) j}{N+1}, & i>j .\end{cases}
$$

Hence we have

$$
\left\|L^{-1}\right\|_{\infty}=\max _{1 \leq i \leq N} \sum_{j=1}^{N}\left|\tilde{l}_{i, j}\right|=\max _{1 \leq i \leq N} \frac{i}{2}(N+1-i) \leq \frac{(N+1)^{2}}{8}
$$

Applying the above estimate we obtain that $\|\widetilde{\mathbf{u}}\|_{\infty} \leq \gamma / 8$. Define $\overline{\mathbf{u}}=\widetilde{\mathbf{u}}$ and $\underline{\mathbf{u}}=-\widetilde{\mathbf{u}}$. We have that for all $\mathbf{w} \in \mathbf{K}(\underline{\mathbf{u}}, \overline{\mathbf{u}})$,

$$
\mathbf{f}(\mathbf{w}, \mathbf{u})-\mathbf{f}(\mathbf{w}, \mathbf{v}) \geq-\left(\frac{\gamma^{2}}{32}+\frac{\gamma(1+\theta)}{8}\right)(\mathbf{u}-\mathbf{v})
$$

whenever $\underline{\mathbf{u}} \leq \mathbf{v} \leq \mathbf{u} \leq \overline{\mathbf{u}}$. Also we have

$$
\left\{\begin{aligned}
\frac{D}{h^{2}} L \overline{\mathbf{u}}=\gamma D \mathbf{z} & \geq\left(\frac{\gamma(1-\theta)^{2}}{32}+\frac{\gamma^{3}}{128}+\frac{\gamma^{2}(1+\theta)}{32}\right) \mathbf{z} \\
& \geq \mathbf{f}(\overline{\mathbf{u}}, \overline{\mathbf{u}})+\left(\frac{\gamma^{2}}{32}+\frac{\gamma(1+\theta)}{8}\right)(\overline{\mathbf{u}}-\underline{\mathbf{u}}), \\
-\frac{D}{h^{2}} L \overline{\mathbf{u}}=-\gamma D \mathbf{z} & \leq\left(-\frac{\gamma(1-\theta)^{2}}{32}-\frac{\gamma^{3}}{128}-\frac{\gamma^{2}(1+\theta)}{32}\right) \mathbf{z} \\
& \leq \mathbf{f}(\underline{\mathbf{u}}, \underline{\mathbf{u}})-\left(\frac{\gamma^{2}}{32}+\frac{\gamma(1+\theta)}{8}\right)(\overline{\mathbf{u}}-\underline{\mathbf{u}}) .
\end{aligned}\right.
$$

Take

$$
C=\left(\frac{\gamma^{2}}{32}+\frac{\gamma(1+\theta)}{8}\right) I
$$

We see from (4.10) and (4.11) that $\overline{\mathbf{u}}$ and $\underline{\mathbf{u}}$ are the coupled upper and lower solutions of (4.4) provided $D>(1-\theta)^{2} / 32$.

Example 2. As a second example we consider the function

$$
f(x, u(x, t), u(x, t-\tau))=\sin (a(x) u(x, t))+\cos (b(x) u(x, t-\tau))
$$


where the function $a(x)$ and $b(x)$ are continuous functions with $|b(x)| \leq b_{0}$ for all $x \in(0,1)$. Since the function $b(x)$ may be arbitrarily oscillate, the monotone property of the function $f(x, u(x, t), u(x, t-\tau))$ in $u(x, t-\tau)$ is usually destroyed.

Assume that $D \geq 3 b_{0} / 8$, and take a positive constant $\mu$ such that

$$
\frac{8}{8 D-b_{0}} \leq \mu \leq \frac{4}{b_{0}}
$$

Define $\underline{\mathbf{u}} \equiv \mathbf{0}$ and $\overline{\mathbf{u}}=\left(\bar{u}_{1}, \bar{u}_{2}, \ldots, \bar{u}_{N}\right)^{T}$ with $\bar{u}_{i}=\mu x_{i}\left(1-x_{i}\right)(i=1,2, \ldots, N)$, where $x_{i}=i h, i=0, \ldots, N+1$, are the mesh points. Then we have that for all $\mathbf{w} \in \mathbf{K}(\underline{\mathbf{u}}, \overline{\mathbf{u}})$,

$$
\mathbf{f}(\mathbf{w}, \mathbf{u})-\mathbf{f}(\mathbf{w}, \mathbf{v}) \geq-b_{0}(\mathbf{u}-\mathbf{v})
$$

whenever $\underline{\mathbf{u}} \leq \mathbf{v} \leq \mathbf{u} \leq \overline{\mathbf{u}}$. Also we have

$$
\left\{\begin{aligned}
\frac{D}{h^{2}} L \overline{\mathbf{u}}=2 \mu D \mathbf{z} & \geq\left(2+\frac{b_{0} \mu}{4}\right) \mathbf{z} \\
& \geq \mathbf{f}(\overline{\mathbf{u}}, \overline{\mathbf{u}})+b_{0}(\overline{\mathbf{u}}-\underline{\mathbf{u}}), \\
\frac{D}{h^{2}} L \underline{\mathbf{u}}=\mathbf{0} & \leq\left(1-\frac{b_{0} \mu}{4}\right) \mathbf{z} \\
& \leq \mathbf{f}(\underline{\mathbf{u}}, \underline{\mathbf{u}})-b_{0}(\overline{\mathbf{u}}-\underline{\mathbf{u}}) .
\end{aligned}\right.
$$

This shows that $\overline{\mathbf{u}}$ and $\underline{\mathbf{u}}$ are the coupled upper and lower solutions of (4.4) with $C=b_{0} I$. Let $\overline{\mathbf{u}}_{n} \equiv \overline{\mathbf{u}}$ and $\underline{\mathbf{u}}_{n} \equiv \underline{\mathbf{u}}(n \geq-s)$. Then the constant sequences $\overline{\mathbf{u}}_{n}$ and $\underline{\mathbf{u}}_{n}$ are the coupled upper and lower solutions of (4.2) with $\bar{C}_{n} \equiv b_{0} I$ provided that $D \geq 3 b_{0} / 8$ and $0 \leq \psi(x, t) \leq \mu x(1-x)$ for all $x \in(0,1)$ and $t \in[-\tau, 0]$. It follows from this construction that the results in Section 3 can be applied to this example.

Example 3. Our final example is for the function

$$
f(x, u(x), u(x, t-\tau))=\mathrm{e}^{b(x) u(x, t-\tau)}
$$

where the function $b(x)$ is a continuous function with $|b(x)| \leq b_{0}$ for all $x \in(0,1)$. As the second example, the monotone property of the function $f(x, u(x, t), u(x, t-\tau))$ in $u(x, t-\tau)$ is usually destroyed because of the arbitrarily oscillate property of the function $b(x)$.

Assume that $D \geq(\sqrt{\mathrm{e}}+1) b_{0} / 4$, and take a positive constant $\zeta$ such that

$$
\frac{\sqrt{\mathrm{e}}+1}{2 D} \leq \zeta \leq \frac{2}{b_{0}}
$$

Define $\underline{\mathbf{u}} \equiv \mathbf{0}$ and $\overline{\mathbf{u}}=\left(\bar{u}_{1}, \bar{u}_{2}, \ldots, \bar{u}_{N}\right)^{T}$ with $\bar{u}_{i}=\zeta x_{i}\left(1-x_{i}\right)(i=1,2, \ldots, N)$, where $x_{i}=i h, i=0, \ldots, N+1$, are the mesh points. Then we have that for all $\mathbf{w} \in \mathbf{K}(\underline{\mathbf{u}}, \overline{\mathbf{u}})$,

$$
\mathbf{f}(\mathbf{w}, \mathbf{u})-\mathbf{f}(\mathbf{w}, \mathbf{v}) \geq-b_{0} \mathrm{e}^{\frac{b_{0} \zeta}{4}}(\mathbf{u}-\mathbf{v})
$$

whenever $\underline{\mathbf{u}} \leq \mathbf{v} \leq \mathbf{u} \leq \overline{\mathbf{u}}$. Since

$$
\left\{\begin{array}{l}
\frac{b_{0} \zeta}{4} \mathrm{e}^{\frac{b_{0} \zeta}{4}} \leq 1 \\
2 D \zeta \geq\left(1+\frac{b_{0} \zeta}{4}\right) \mathrm{e}^{\frac{b_{0} \zeta}{4}}
\end{array}\right.
$$


we have

$$
\left\{\begin{aligned}
\frac{D}{h^{2}} L \overline{\mathbf{u}}=2 D \zeta \mathbf{z} & \geq\left(\mathrm{e}^{\frac{b_{0} \zeta}{4}}+\frac{b_{0} \zeta}{4} \mathrm{e}^{\frac{b_{0} \zeta}{4}}\right) \mathbf{z} \\
& \geq \mathbf{f}(\overline{\mathbf{u}}, \overline{\mathbf{u}})+b_{0} \mathrm{e}^{\frac{b_{0} \zeta}{4}}(\overline{\mathbf{u}}-\underline{\mathbf{u}}), \\
\frac{D}{h^{2}} L \underline{\mathbf{u}}=\mathbf{0} & \leq\left(1-\frac{b_{0} \zeta}{4} \mathrm{e}^{\frac{b_{0} \zeta}{4}}\right) \mathbf{z} \\
& \leq \mathbf{f}(\underline{\mathbf{u}}, \underline{\mathbf{u}})-b_{0} \mathrm{e}^{\frac{b_{0} \zeta}{4}}(\overline{\mathbf{u}}-\underline{\mathbf{u}}) .
\end{aligned}\right.
$$

This shows that $\overline{\mathbf{u}}$ and $\underline{\mathbf{u}}$ are the coupled upper and lower solutions of (4.4) with $C=b_{0} \mathrm{e}^{\frac{b_{0} \zeta}{4}} I$. As before, the constant sequences $\overline{\mathbf{u}}_{n} \equiv \overline{\mathbf{u}}$ and $\underline{\mathbf{u}}_{n} \equiv \underline{\mathbf{u}}(n \geq-s)$ are the coupled upper and lower solutions of (4.2) with $C_{n} \equiv b_{0} \mathrm{e}^{\frac{b_{0} \zeta}{4}} I$ provided that $D \geq(\sqrt{\mathrm{e}}+1) b_{0} / 4$ and $0 \leq \psi(x, t) \leq \zeta x(1-x)$ for all $x \in(0,1)$ and $t \in[-\tau, 0]$. Hence, the results in Section 3 can be applied to this example.

\subsection{Numerical results for Example 2}

We consider the problem (4.1) where the function $f$ is given in (4.12). The physical parameters and the initial function are taken as $D=1, \tau=1, a(x) \equiv 1, b(x)=\sin \left(x-\frac{1}{8}\right)$, and $\psi(x, t) \equiv 0$. Define $\underline{\mathbf{u}} \equiv \mathbf{0}$ and $\overline{\mathbf{u}}=\left(\bar{u}_{1}, \bar{u}_{2}, \ldots, \bar{u}_{N}\right)^{T}$ with $\bar{u}_{i}=3 x_{i}\left(1-x_{i}\right)(i=1,2, \ldots, N)$, where $x_{i}=i h, i=0, \ldots, N+1$, are the mesh points. From the above construction process, we have that $\overline{\mathbf{u}}$ and $\underline{\mathbf{u}}$ are the coupled upper and lower solutions of (4.4) with $C=\frac{7}{8} I$. Moreover, the constant sequences $\overline{\mathbf{u}}_{n} \equiv \overline{\mathbf{u}}$ and $\underline{\mathbf{u}}_{n} \equiv \underline{\mathbf{u}}(n \geq-s)$ are the coupled upper and lower solutions of (4.2). Take mesh size as $h=k=0.05$. By the iterative scheme (2.7) in [12], we compute the maximal sequence $\left\{\overline{\mathbf{u}}_{n}^{(m)}\right\}$ and the minimal sequence $\left\{\underline{\mathbf{u}}_{n}^{(m)}\right\}$ for the above test problem with $\overline{\mathbf{u}}_{n}^{(0)} \equiv \overline{\mathbf{u}}, \underline{\mathbf{u}}_{n}^{(0)} \equiv \underline{\mathbf{u}}, \Gamma_{n} \equiv I$, and $\underline{\Psi}_{n}=\mathbf{0}$. In the iteration process the stopping criterion is determined by $\left\|\mathbf{u}_{n}^{(m+1)}-\mathbf{u}_{n}^{(m)}\right\|_{\infty} \leq \varepsilon$ for various values of $\varepsilon$, where $\mathbf{u}_{n}^{(m)}$ represents either $\overline{\mathbf{u}}_{n}^{(m)}$ or $\underline{\mathbf{u}}_{n}^{(m)}$. To count the number of iterations, we let $\varepsilon=10^{-5}$ and $10^{-8}$. Our computations for $n$ up to 1000 show that the numbers of iterations are same at every $n$ for each of the sequences $\left\{\overline{\mathbf{u}}_{n}^{(m)}\right\}$ and $\left\{\underline{\mathbf{u}}_{n}^{(m)}\right\}$. When $\varepsilon=10^{-5}$, the number of iterations is 5 for both the sequences $\left\{\overline{\mathbf{u}}_{n}^{(m)}\right\}$ and $\left\{\underline{\mathbf{u}}_{n}^{(m)}\right\}$. When $\varepsilon$ is taken as $10^{-8}$, the number of iterations is increased to 8 for the sequence $\left\{\overline{\mathbf{u}}_{n}^{(m)}\right\}$ and to 7 for the sequence $\left\{\underline{\mathbf{u}}_{n}^{(m)}\right\}$. In the computations, we also find that the maximal sequence $\left\{\overline{\mathbf{u}}_{n}^{(m)}\right\}$ and the minimal sequence $\left\{\underline{\mathbf{u}}_{n}^{(m)}\right\}$ tend to the same limit $\mathbf{u}_{n}^{*}$. Numerical results for the values of these sequences at $n=10$ are given in Table 1. By Theorem 2.1 in [12], the limit $\mathbf{u}_{n}^{*}$ is the unique solution of $(4.2)$ in $\mathbf{K}(\underline{\mathbf{u}}, \overline{\mathbf{u}})$. We choose $\overline{\mathbf{u}}_{n}^{(m)}$ as the computed solution $\mathbf{u}_{n}^{*}$ when $\left\|\overline{\mathbf{u}}_{n}^{(m)}-\overline{\mathbf{u}}_{n}^{(m-1)}\right\|_{\infty} \leq 10^{-8}$. The number of iterations for each of $\mathbf{u}_{n}^{*}(1 \leq n \leq 1000)$ is 8 . The values of the solution $\mathbf{u}_{n}^{*}$ are given in Table 2 .

Next by the iterative scheme (3.3) of this paper, we compute the sequences $\left\{\overline{\mathbf{u}}^{(m)}\right\}$ and $\left\{\underline{\mathbf{u}}^{(m)}\right\}$ for the above test problem with $\overline{\mathbf{u}}^{(0)}=\overline{\mathbf{u}}, \underline{\mathbf{u}}^{(0)}=\underline{\mathbf{u}}, C=\frac{7}{8} I$ and $\Gamma^{*}=I$. The stopping criterion is determined by $\left\|\overline{\mathbf{u}}^{(m)}-\underline{\mathbf{u}}^{(m)}\right\|_{\infty} \leq \varepsilon$ for various values of $\varepsilon$. When $\varepsilon=10^{-5}$, the number of iterations is 11 , and it is increased to 17 if $\varepsilon$ is taken as $10^{-8}$. In our numerical computations, the monotone nonincreasing convergence property of $\left\{\overline{\mathbf{u}}^{(m)}\right\}$ and the monotone nondecreasing convergence property of $\left\{\underline{\mathbf{u}}^{(m)}\right\}$ are observed. Moreover, they have the same limit $\mathbf{u}^{*}$. By Theorem 3.1 of this paper, the limit $\mathbf{u}^{*}$ is the unique solution of (4.4) in $\mathbf{K}(\underline{\mathbf{u}}, \overline{\mathbf{u}})$. Numerical results for the monotone property of these sequences are given in Table 3 . These results are also sketched in Figure 1. When $\left\|\overline{\mathbf{u}}^{(m)}-\underline{\mathbf{u}}^{(m)}\right\|_{\infty} \leq 10^{-8}$, we take $\overline{\mathbf{u}}^{(m)}$ as the computed solution $\mathbf{u}^{*}$. The number of iterations for $\mathbf{u}^{*}$ is 17 . The values of $\mathbf{u}^{*}$ are listed in Table 2 .

It is seen from Table 2 that the difference $\left\|\mathbf{u}_{n}^{*}-\mathbf{u}^{*}\right\|_{\infty}$ is less than $7 \times 10^{-5}$ when $n$ is between 25 and 31 , and this difference is reduced to $10^{-5}$ at $n=32$. Indeed, we find in the computations that the sequence $\left\{\mathbf{u}_{n}^{*}\right\}$ 
TABLE 1. (a) The maximal sequence $\left\{\overline{\mathbf{u}}_{n}^{(m)}\right\}$ (at $n=10$ ); (b) the minimal sequence $\left\{\underline{\mathbf{u}}_{n}^{(m)}\right\}$ (at $n=10)$.

(a)

\begin{tabular}{cccccccccc}
\hline & $\bar{u}_{1,10}^{(m)}$ & $\bar{u}_{2,10}^{(m)}$ & $\bar{u}_{3,10}^{(m)}$ & $\bar{u}_{4,10}^{(m)}$ & $\bar{u}_{5,10}^{(m)}$ & $\bar{u}_{6,10}^{(m)}$ & $\bar{u}_{7,10}^{(m)}$ & $\bar{u}_{8,10}^{(m)}$ & $\bar{u}_{9,10}^{(m)}$ \\
\hline$m=1$ & 0.03194 & 0.06109 & 0.08721 & 0.11011 & 0.12967 & 0.14578 & 0.15837 & 0.16739 & 0.17282 \\
$m=2$ & 0.02588 & 0.04920 & 0.06991 & 0.08794 & 0.10327 & 0.11586 & 0.12568 & 0.13271 & 0.13693 \\
$m=3$ & 0.02551 & 0.04846 & 0.06882 & 0.08654 & 0.10159 & 0.11394 & 0.12357 & 0.13046 & 0.13460 \\
$m=4$ & 0.02548 & 0.04841 & 0.06875 & 0.08645 & 0.10148 & 0.11381 & 0.12343 & 0.13031 & 0.13444 \\
$m=5$ & 0.02548 & 0.04841 & 0.06874 & 0.08644 & 0.10147 & 0.11380 & 0.12342 & 0.13030 & 0.13443 \\
\hline
\end{tabular}

(b)

\begin{tabular}{cccccccccc}
\hline & $\underline{u}_{1,10}^{(m)}$ & $\underline{u}_{2,10}^{(m)}$ & $\underline{u}_{3,10}^{(m)}$ & $\underline{u}_{4,10}^{(m)}$ & $\underline{u}_{5,10}^{(m)}$ & $\underline{u}_{6,10}^{(m)}$ & $\underline{u}_{7,10}^{(m)}$ & $\underline{u}_{8,10}^{(m)}$ & $\underline{u}_{9,10}^{(m)}$ \\
\hline$m=1$ & 0.02401 & 0.04553 & 0.06454 & 0.08103 & 0.09501 & 0.10646 & 0.11537 & 0.12174 & 0.12556 \\
$m=2$ & 0.02539 & 0.04823 & 0.06847 & 0.08609 & 0.10105 & 0.11333 & 0.12290 & 0.12974 & 0.13386 \\
$m=3$ & 0.02547 & 0.04840 & 0.06872 & 0.08642 & 0.10144 & 0.11377 & 0.12339 & 0.13026 & 0.13440 \\
$m=4$ & 0.02548 & 0.04841 & 0.06874 & 0.08644 & 0.10147 & 0.11380 & 0.12342 & 0.13030 & 0.13443 \\
\hline
\end{tabular}

TABLE 2. The solution $\mathbf{u}_{n}^{*}$ of (4.2) and the solution $\mathbf{u}^{*}$ of (4.4).

\begin{tabular}{cccccccccc}
\hline & $i=2$ & $i=4$ & $i=6$ & $i=8$ & $i=10$ & $i=12$ & $i=14$ & $i=16$ & $i=18$ \\
\hline$u_{i, 25}^{*}$ & 0.04953 & 0.08856 & 0.11672 & 0.13373 & 0.13940 & 0.13371 & 0.11669 & 0.08854 & 0.04951 \\
$u_{i, 26}^{*}$ & 0.04952 & 0.08856 & 0.11672 & 0.13372 & 0.13939 & 0.13370 & 0.11668 & 0.08853 & 0.04951 \\
$u_{i, 27}^{*}$ & 0.04952 & 0.08855 & 0.11671 & 0.13371 & 0.13938 & 0.13368 & 0.11667 & 0.08852 & 0.04950 \\
$u_{i, 28}^{*}$ & 0.04952 & 0.08855 & 0.11670 & 0.13370 & 0.13937 & 0.13367 & 0.11666 & 0.08851 & 0.04950 \\
$u_{i, 29}^{*}$ & 0.04952 & 0.08855 & 0.11670 & 0.13369 & 0.13936 & 0.13367 & 0.11666 & 0.08851 & 0.04950 \\
$u_{i, 30}^{*}$ & 0.04952 & 0.08854 & 0.11669 & 0.13369 & 0.13936 & 0.13366 & 0.11665 & 0.08850 & 0.04949 \\
$u_{i, 31}^{*}$ & 0.04951 & 0.08854 & 0.11669 & 0.13368 & 0.13935 & 0.13365 & 0.11665 & 0.08850 & 0.04949 \\
$u_{i, 32}^{*}$ & 0.04951 & 0.08854 & 0.11669 & 0.13368 & 0.13935 & 0.13365 & 0.11664 & 0.08850 & 0.04949 \\
$u_{i}^{*}$ & 0.04951 & 0.08853 & 0.11668 & 0.13367 & 0.13934 & 0.13364 & 0.11663 & 0.08849 & 0.04949 \\
\hline
\end{tabular}

tends to $\mathbf{u}^{*}$ as $n \rightarrow \infty$. Figure 2 shows the asymptotic convergence property of the solution $\mathbf{u}_{n}^{*}$ as $n \rightarrow \infty$. This coincides with the result in Theorem 3.3 or Corollary 3.1 .

From Figures 1 and 2, it seems that the solutions $\mathbf{u}_{n}^{*}$ and $\mathbf{u}^{*}$ are symmetric along the $x$-direction. In fact, we see from Table 2 that they are almost symmetric without being really symmetric.

Acknowledgements. The author is very grateful to the referees for their useful comments and suggestions. 
TABLE 3. (a) Monotone property of the sequence $\left\{\overline{\mathbf{u}}^{(m)}\right\}$; (b) monotone property of the sequence $\left\{\underline{\mathbf{u}}^{(m)}\right\}$.

(a)

\begin{tabular}{cccccccccc}
\hline & $\bar{u}_{1}^{(m)}$ & $\bar{u}_{2}^{(m)}$ & $\bar{u}_{3}^{(m)}$ & $\bar{u}_{4}^{(m)}$ & $\bar{u}_{5}^{(m)}$ & $\bar{u}_{6}^{(m)}$ & $\bar{u}_{7}^{(m)}$ & $\bar{u}_{8}^{(m)}$ & $\bar{u}_{9}^{(m)}$ \\
\hline$m=1$ & 0.05337 & 0.10335 & 0.14915 & 0.19010 & 0.22562 & 0.25525 & 0.27860 & 0.29540 & 0.30546 \\
$m=2$ & 0.03415 & 0.06553 & 0.09388 & 0.11896 & 0.14056 & 0.15847 & 0.17256 & 0.18269 & 0.18879 \\
$m=3$ & 0.02863 & 0.05462 & 0.07787 & 0.09825 & 0.11567 & 0.13004 & 0.14129 & 0.14936 & 0.15421 \\
$m=4$ & 0.02689 & 0.05120 & 0.07284 & 0.09174 & 0.10784 & 0.12109 & 0.13144 & 0.13885 & 0.14330 \\
$m=5$ & 0.02633 & 0.05008 & 0.07119 & 0.08961 & 0.10528 & 0.11816 & 0.12821 & 0.13541 & 0.13973 \\
$m=6$ & 0.02614 & 0.04970 & 0.07064 & 0.08890 & 0.10442 & 0.11718 & 0.12731 & 0.13426 & 0.13854 \\
\hline
\end{tabular}

(b)

\begin{tabular}{lccccccccc}
\hline & $\underline{u}_{1}^{(m)}$ & $\underline{u}_{2}^{(m)}$ & $\underline{u}_{3}^{(m)}$ & $\underline{u}_{4}^{(m)}$ & $\underline{u}_{5}^{(m)}$ & $\underline{u}_{6}^{(m)}$ & $\underline{u}_{7}^{(m)}$ & $\underline{u}_{8}^{(m)}$ & $\underline{u}_{9}^{(m)}$ \\
\hline$m=1$ & 0.01194 & 0.02173 & 0.02966 & 0.03600 & 0.04098 & 0.04480 & 0.04760 & 0.04952 & 0.05063 \\
$m=2$ & 0.02021 & 0.03801 & 0.05346 & 0.06667 & 0.07770 & 0.08662 & 0.09350 & 0.09837 & 0.10128 \\
$m=3$ & 0.02386 & 0.04520 & 0.06403 & 0.08034 & 0.09413 & 0.10541 & 0.11417 & 0.12043 & 0.12418 \\
$m=4$ & 0.02526 & 0.04797 & 0.06809 & 0.08560 & 0.10045 & 0.11264 & 0.12213 & 0.12892 & 0.13300 \\
$m=5$ & 0.02576 & 0.04897 & 0.06956 & 0.08750 & 0.10274 & 0.11526 & 0.12502 & 0.13200 & 0.13619 \\
$m=6$ & 0.02594 & 0.04932 & 0.07008 & 0.08817 & 0.10355 & 0.11618 & 0.12604 & 0.13309 & 0.13732 \\
\hline
\end{tabular}
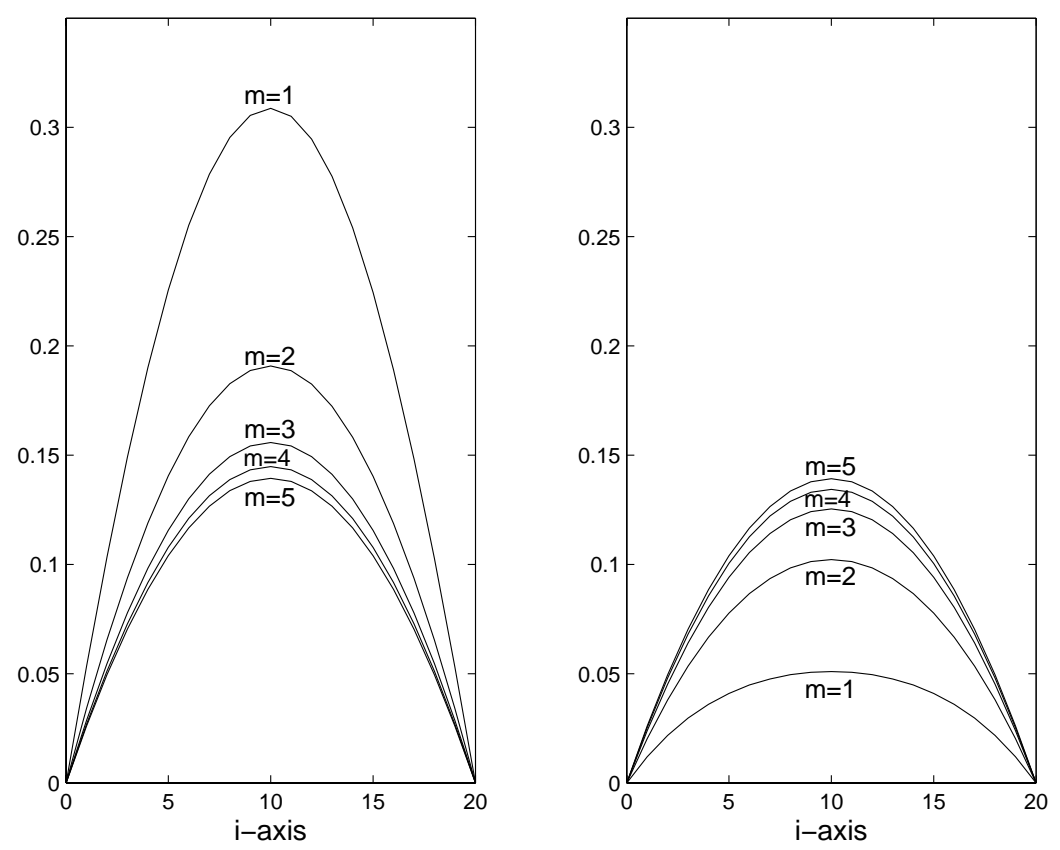

FigurE 1. The monotone property of the sequences $\left\{\overline{\mathbf{u}}^{(m)}\right\}$ and $\left\{\underline{\mathbf{u}}^{(m)}\right\}$. (Left: iterative values $\bar{u}_{i}^{(m)}$; right: iterative values $\underline{u}_{i}^{(m)}$.) 


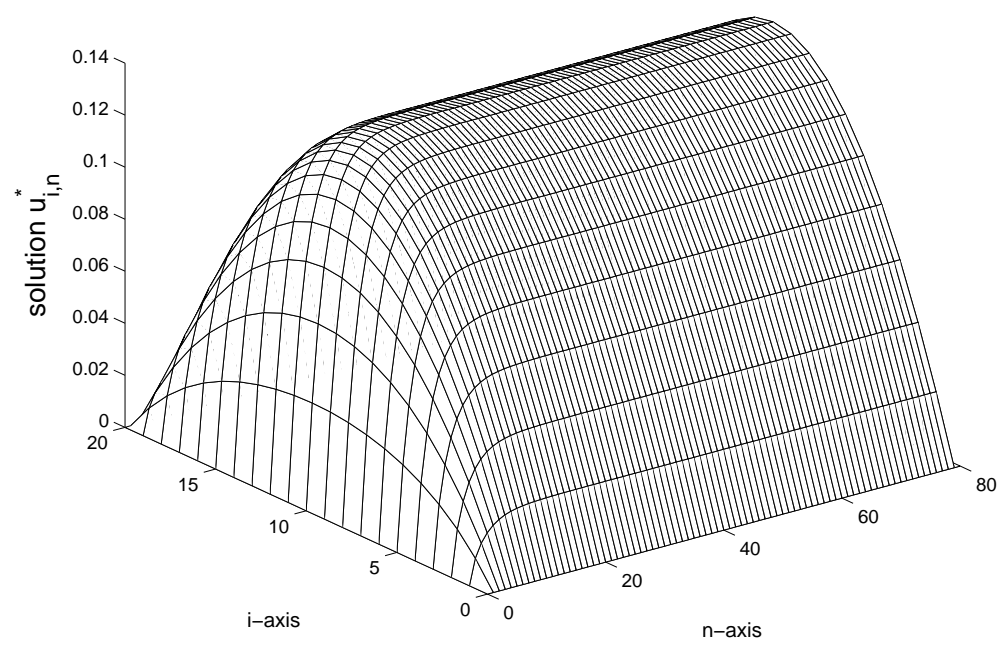

Figure 2. Asymptotic behavior of the solution $\mathbf{u}_{n}^{*}$ as $n \rightarrow \infty$.

\section{REFERENCES}

[1] W.F. Ames, Numerical Methods for Partial Differential Equations. 3rd ed., Academic Press, San Diego (1992).

[2] D.G. Aronson and H.F. Weinberger, Nonlinear diffusion in population genetics, combustion and nerve propagation. Lecture Notes in Math. 446 (1975) 5-49.

[3] A. Berman and R. Plemmons, Nonnegative Matrix in the Mathematical Science. Academic Press, New York (1979).

[4] E.D. Conway, D. Hoff and J.A. Smoller, Large time behavior of solutions of systems of nonlinear reaction-diffusion equations. SIAM J. Math. Appl. 35 (1978) 1-16.

[5] G.E. Forsythe and W.R. Wasow, Finite Difference Methods for Partial Differential Equations. John Wiley, New York (1964).

[6] Y. Hamaya, On the asymptotic behavior of a diffusive epidemic model (AIDS). Nonlinear Anal. 36 (1999) 685-696.

[7] A.W. Leung and D. Clark, Bifurcation and large time asymptotic behavior for prey-predator reaction-diffusion equations with Dirichlet boundary data. J. Differential Equations 25 (1980) 113-127.

[8] X. Lu, Persistence and extinction in a competition-diffusion system with time delays. Canad. Appl. Math. Quart. 2 (1994) $231-246$.

[9] J.M. Ortega and W.C. Rheinboldt, Iterative Solution of Nonlinear Equations in Several Variables. Academic Press, New York (1976).

[10] C.V. Pao, Asymptotic behavior of solutions for finite-difference equations of reaction-diffusion. J. Math. Anal. Appl. 144 (1989) 206-225.

[11] C.V. Pao, Dynamics of a finite difference system of reaction diffusion equations with time delay. J. Differ. Equations Appl. 4 (1998) 1-11.

[12] C.V. Pao, Monotone iterations for numerical solutions of reaction-diffusion-convection equations with time delay. Numer. Methods Partial Differential Equations 14 (1998) 339-351.

[13] C.V. Pao, Monotone methods for a finite difference system of reaction diffusion equation with time delay. Comput. Math. Appl. 36 (1998) 37-47.

[14] C.V. Pao, Nonlinear Parabolic and Elliptic Equations. Plenum Press, New York (1992).

[15] C.V. Pao, Numerical methods for coupled systems of nonlinear parabolic boundary value problems. J. Math. Anal. Appl. 151 (1990) 581-608.

[16] C.V. Pao, Numerical methods for systems of nonlinear parabolic equations with time delays. J. Math. Anal. Appl. 240 (1999) 249-279.

[17] R.S. Varge, Matrix Iterative Analysis. Prentice-Hall, Englewood Cliffs, NJ (1962).

[18] Y. Yamada, Asymptotic behavior of solutions for semilinear Volterra diffusion equations. Nonlinear Anal. 21 (1993) $227-239$.

[19] Z.P. Yang and C.V. Pao, Positive solutions and dynamics of some reaction diffusion models in HIV transmission. Nonlinear Anal. 35 (1999) 323-341. 